\title{
Elastic crystals with a triple point
}

\author{
L. Truskinovsky*, G. Zanzotto** \\ * Department of Aerospace Engineering and Mechanics, University of Minnesota \\ 110 Union St. SE, Minneapolis MN 55455, USA; trusk@ aem.umn.edu \\ ** Dipartimento di Metodi e Modelli Matematici per le Scienze Applicate (DMMMSA), \\ Università di Padova, Via Belzoni 7, 35131 Padova, Italy; zanzotto@dmsa.unipd.it
}

\begin{abstract}
The peculiar behavior of active crystals is due to the presence of evolving phase mixtures the variety of which depends on the number of coexisting phases and the multiplicity of symmetryrelated variants. According to Gibbs' phase rule, the number of phases in a single-component crystal is maximal at a triple point in the $p-T$ phase diagram. In the vicinity of this special point the number of metastable twinned microstructures will also be the highest - a desired effect for improving performance of smart materials. To illustrate the complexity of the energy landscape in the neighborhood of a triple point, and to produce a workable example for numerical simulations, in this paper we construct a generic Landau strain-energy function for a crystal with the coexisting tetragonal (t), orthorhombic (o), and monoclinic $(\mathrm{m})$ phases. As a guideline, we utilize the experimental observations and crystallographic data on the t-o-m transformations of zirconia $\left(\mathrm{ZrO}_{2}\right)$, a major toughening agent for ceramics. After studying the kinematics of the t-o-m phase transformations, we re-evaluate the available experimental data on zirconia polymorphs, and propose a new mechanism for the technologically important $\mathrm{t}-\mathrm{m}$ transition. In particular, our proposal entails the softening of a different tetragonal modulus from the one previously considered in the literature. We derive the simplest expression for the energy function for a t-o-m crystal with a triple point as the lowest-order polynomial in the relevant strain components, exhibiting the complete set of wells associated with the t-o-m phases and their symmetry-related variants. By adding the potential of a hydrostatic loading, we study the $p-T$ phase diagram and the energy landscape of our crystal in the vicinity of the t-o-m triple point. We show that the simplest assumptions concerning the order-parameter coupling and the temperature dependence of the Landau coefficients produce a phase diagram that is in good qualitative agreement with the experimental diagram of $\mathrm{ZrO}_{2}$.
\end{abstract}

Key words: A) Phase transformations, triple point ; B) Crystalline material, Zirconia $\left(\mathrm{ZrO}_{2}\right)$; C) Energy methods, phase diagrams 


\section{Introduction}

Symmetry-breaking martensitic transformations are at the origin of the peculiar macroscopic properties of active crystalline materials, such as shape-memory alloys and transformationtoughened ceramics. It has been recognized that a detailed understanding of the transformation mechanisms and the associated optimal microstructures is essential for the control, improvement and design of the new structures and devices in which these active materials are utilized.

Since diffusion and ordering do not play a major role in most applications of smart materials, their macroscopic behavior can be modeled at a phenomenological level within the framework of nonlinear elasticity theory. The constitutive aspect of such modeling requires giving an explicit expression for the free energy density of the material, which typically is a non-quasi-convex function of the strain variables and, possibly, other order parameters. A comprehensive study of the temperature- and strain-dependent energy functions suitable for transforming crystals was initiated by Landau and his school (see [IS90, TD96] for recent reviews). A more recent line of investigation started with the works of Ericksen (e.g. [E70, E80, E93]) who has brought this approach within the agenda of nonlinear continuum mechanics, connecting it, on the one hand, to the "crystallographic theory of martensite' used in metallurgy and materials science (e.g. [WLR53, W64, Chr75, $\mathrm{R} 78, \mathrm{Kh} 83]$ ), and, on the other hand, to non-convex variational calculus (e.g. [BJ87, CK88, BJ92, BFJK94, M98]). In the last years thermoelasticity theory has proven quite successful in modeling symmetry-breaking transformations that involve finite but not too-large lattice distortions (see [Lu96, JH00, BJ00, PZ00] for recent reviews).

Crystals with only two phases are typically considered in the models; a general analysis of the two-phase bifurcations in simple Bravais lattices can be found in [E93, PZ00]. Active materials, however, often exhibit more than two polymorphs. In fact, according to Gibbs' phase rule, even a single-component system may admit three equally stable phases at an isolated 'triple point' in its pressure-temperature phase diagram (e.g. [DF80]). Due to the large number of energy wells associated with the phase variants in the vicinity of a such a triple point, the crystal may have a significantly richer class of equilibrium microstructures, and therefore a higher flexibility in accommodating imposed loads. Since this property is crucial in numerous applications of smart materials, the constitutive investigation in the neighborhood of a triple point presents particular interest (e.g. [WP98]).

In this paper we construct an explicit expression for the free energy function of a crystal with tetragonal (t), orthorhombic (o), and monoclinic $(\mathrm{m})$ phases. Polymorphs with these symmetries are observed in many materials, for instance in various transition-metal oxides. Here we focus on the constitutive modeling of zirconia $\left(\mathrm{ZrO}_{2}\right)$, the main toughening agent in transformation-toughened ceramics. In the applications, small zirconia inclusions in an inert ceramic matrix are used to control and enhance the otherwise low ductility of the ceramic composites (see for instance [GHS89]). The experimental phase diagram of zirconia for not too-high pressures and temperatures is shown in Fig. 1. This diagram, which contains a t-o-m triple point, is fairly well established, ${ }^{1}$ although the actual mechanism of the t-o-m transformations in $\mathrm{ZrO}_{2}$ remains somewhat obscure.

\footnotetext{
${ }^{1}$ See for instance [LTAP93], [OM98], and the references quoted therein. A point of some uncertainty in Fig. 1 is the slope of the t-o boundary, which is reported as negative in one study [BJP95].
} 


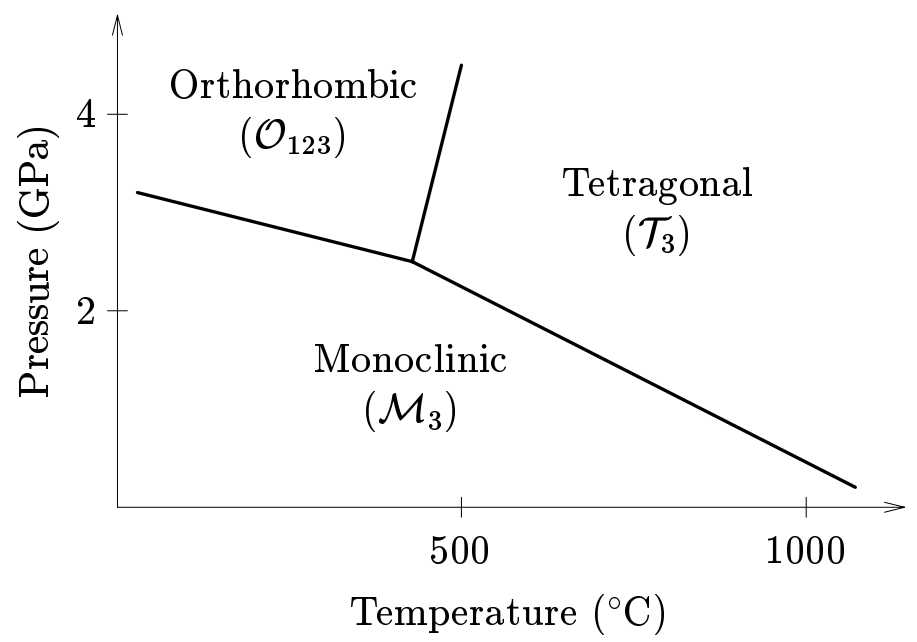

Fig. 1 Portion of the experimental phase diagram for zirconia exhibiting the tetragonal-orthorhombicmonoclinic (t-o-m) triple point. The point groups of the phases are specified in Section 3. The corresponding symmetry-breaking strains are given in Fig. 4.

Previous attempts to model the t-m zirconia transformation within the context of nonlinear elasticity are reviewed in [BHL83, Cha88, BT93, ST96]; some applications of the non-hydrostatic phase diagrams for transformation toughening can be found in [ST96] and the literature quoted therein. In the present paper we revisit the problem by first examining the potential mechanisms of the pertinent t-o-m transitions. The knowledge of the kinematic path of a phase change is crucial for writing the correct energy function and for establishing the metastability ranges in the equilibrium phase diagram. Our kinematic analysis of the t-om symmetry-breaking mechanisms derived from [Z96b] (see also [BLL99, PZ00]), and the available experimental data on zirconia polymorphs, suggest a $\mathrm{t}-\mathrm{m}$ transformation path that is distinct from the one previously considered in the literature; in particular it entails the softening of a different tetragonal modulus.

The $\mathrm{t}-\mathrm{m}$ transformation path considered so far originates from the instability induced by the (partial) softening of the tetragonal modulus $C_{44}$ (e.g. [Cha88]). This mechanism was adopted by [BT93, ST96], who constructed the corresponding energy function and investigated the distortion of the $p$ - $T$ phase diagram under the influence of non-hydrostatic stresses. In spite of the internal consistency of the corresponding model, the t-o orientation relationships reported experimentally for zirconia by [SMS74, BFV91] indicate that the $C_{44}$ related mechanism may not be the one at work in $\mathrm{ZrO}_{2}$. What is perhaps more important, this mechanism does not allow for the presence of orthorhombic zirconia, and, ultimately, of the t-o-m triple point, in the phase diagram of the crystal. On the contrary, the existence of an orthorhombic polymorph is a natural consequence of the softening of the tetragonal moduli $C_{11}-C_{12}$ or $C_{66}$. Indeed, the corresponding bifurcations do not lead directly to structures with monoclinic symmetry: each one describes the formation of an orthorhombic phase with distinct centering. The o-m transformation then results from a second bifurcation taking place after the t-o symmetry breaking; the well-known first-order t-m transformation of zirconia thus originates from this underlying 'double-bifurcation' mechanism. The 
analysis of the available data on orthorhombic $\mathrm{ZrO}_{2}$ suggests that $C_{11}-C_{12}$ should be the modulus whose softening triggers the t-o bifurcation.

With this two-stage mechanism in mind, we construct an energy function as the lowest-order polynomial in finite strains capable of exhibiting a suitable t-o-m triple point. In the present model, which treats the zirconia crystal as a simple lattice without an internal motif, ${ }^{2}$ different strain order parameters are responsible for the t-o and o-m transformations. The final form of our Landau energy is a sixth-degree polynomial exhibiting the complete set of wells associated with the t-o-m phases and their symmetry-related variants, one of the several formal possibilities considered in [SD86, TD96]. By adding the potential of a hydrostatic loading, we study the $p-T$ phase diagram of the crystal and its energy landscape near the triple point. Although at this stage we do not attempt the optimal fit of any material parameters, the resulting phase diagram in Fig. 7 is in good qualitative agreement with the experimental one in Fig. 1.

This paper is organized as follows. In Section 2 we study the kinematics of the t-o-m phase transformations, describing the complete set of possible t-o-m symmetry-breaking mechanisms. In Section 3 we focus on the crystalline structures of zirconia, and critically evaluate the existing experimental information concerning its orientation relationships, transformation strains, and phase diagram, against the kinematical results of Section 2 . Based on this analysis, we suggest a new t-m transformation mechanism for $\mathrm{ZrO}_{2}$, and specify the corresponding order parameters. In Section 4 we write the simplest polynomial energy with tetragonal symmetry and minimal order parameter coupling, whose minimizers under varying temperature and pressure describe the (meta)stable configurations with the correct t-o-m symmetries. In Section 5 we study the relative stability of the t-o-m phases and specify their coexistence domains. Then, in an explicit example, we compute the phase diagram indicating the stability boundaries of the different phases, the corresponding Maxwell lines, and the t-o-m triple point. In the last Section we discuss some possible applications of our results and indicate some open problems.

\section{Kinematics of the tetragonal-orthorhombic-monoclinic (t-o-m) crystals}

We study the phase transitions of a crystal whose high-symmetry parent phase is given by a primitive tetragonal Bravais lattice:

$$
\Lambda\left(\boldsymbol{t}_{a}\right)=\left\{\boldsymbol{x}=\sum M_{a} \boldsymbol{t}_{a}, M_{a} \text { integers, } a=1,2,3\right\} .
$$

The three mutually orthogonal independent vectors $\boldsymbol{t}_{a}$ are shown in Fig. 2, with the corresponding orthonormal basis $\boldsymbol{c}_{a}$. In what follows we express any tensors through their representative matrices in the basis $\boldsymbol{c}_{a}$, which provides the standard crystallographic indices

\footnotetext{
${ }^{2}$ We remark that in all the above-mentioned models the zirconia crystal is regarded as a simple Bravais lattice (called the 'skeletal lattice'). In this approximation one disregards the atoms within the unit cell of the skeletal lattice (these atoms form the 'motif' of the crystal). Equivalently, following Born's hypothesis ([B15], [BH54], [St50]), the motif atoms are assumed to be (istantaneously) equilibrated within the skeletal structure. Energy functions utilizing motif variables as order parameters have been proposed in the literature for some of the zirconia transformations (not involving the t-o-m triple point), but have not been studied in detail (see for instance [ID89, FPF00]).
} 
for tetragonal lattices. The point group of the lattice $\Lambda\left(t_{a}\right)$ is denoted by $T_{3}$ (in all the point groups we only list the elements with positive determinant):

$$
T_{3}=\left\{1, \quad \boldsymbol{R}_{\boldsymbol{c}_{1}}{ }^{\pi}, \boldsymbol{R}_{\boldsymbol{c}_{2}}{ }^{\pi}, \boldsymbol{R}_{\boldsymbol{c}_{3}}{ }^{\pi}, \boldsymbol{R}_{\boldsymbol{c}_{1}+\boldsymbol{c}_{2}}{ }^{\pi}, \boldsymbol{R}_{\boldsymbol{c}_{1}-\boldsymbol{c}_{2}}{ }^{\pi}, \boldsymbol{R}_{\boldsymbol{c}_{3}}{ }^{\pi / 2}, \boldsymbol{R}_{\boldsymbol{c}_{3}}^{3 \pi / 2}\right\}
$$

Here $\boldsymbol{R}_{\boldsymbol{k}}{ }^{\psi}$ denotes a rotation of angle $\psi$ about the axis $\boldsymbol{k}$. The elements of the group $T_{3}$ indicate that the lattice has a four-fold symmetry axis along the unit vector $\boldsymbol{c}_{3}$, and four twofold axes along the directions $\boldsymbol{c}_{1}, \boldsymbol{c}_{2}, \boldsymbol{c}_{1}+\boldsymbol{c}_{2}$, and $\boldsymbol{c}_{1}-\boldsymbol{c}_{2}$.

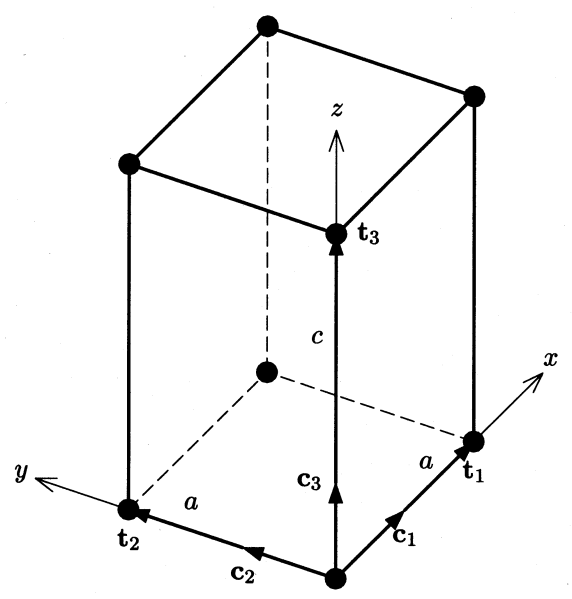

Fig. 2. The primitive tetragonal reference cell used to describe the deformations of the t-o-m lattices. The reference basis $t_{a}$ is shown, where $t_{1}$ and $t_{2}$ have equal length, and the $t_{a}$ are mutually orthogonal. (the four-fold tetragonal axis is along $\boldsymbol{t}_{3}$ ). Also shown is the associated orthonormal basis $\boldsymbol{c}_{a}$, used for representing any tensors as matrices.

We assume that the symmetry-breaking phase changes involve the smallest stretches that produce the observed low-symmetry lattices, that is, we assume the t-o-m transformations are produced by lattice distortions that are close enough to the identity tensor 1 ('weak transformations', in the terminology of [E89]). The energy function we seek will then be defined in a neighborhood $N$ of $\mathbf{1}$ in the six-dimensional space of all the symmetric, positive-definite tensors. Understanding how the 'small' stretches in $N$ act to deform the reference tetragonal basis $\boldsymbol{t}_{a}$ provides important information on the kinematics of $\mathrm{t}-\mathrm{o}-\mathrm{m}$ phase changes.

We choose $N$ to be tetragonally invariant (that is, if $\boldsymbol{U} \in N$, then $\boldsymbol{R}^{t} \boldsymbol{U} \in N$ for all $\boldsymbol{R} \in$ $T_{3}$ ), and sufficiently small. Then, if a stretch $\boldsymbol{U} \in N$ is applied to $\boldsymbol{t}_{a}$ we obtain a basis whose point group is equal to, or is a subgroup of, the point group (2.2) - see for instance [E80, P84, BJ92, PZ00]. This means that we can analyze the t-o-m symmetry-breaking processes by studying the group-subgroup relations for the point groups contained in $T_{3}$. A stretch $\boldsymbol{U}$ in $N$ is called 'symmetry preserving' for $\boldsymbol{t}_{a}$ if the point group of the deformed basis $\boldsymbol{U} \boldsymbol{t}_{a}$ is $T_{3} ; \boldsymbol{U}$ is called 'symmetry breaking' if the point group of $\boldsymbol{U} \boldsymbol{t}_{a}$ is a subgroup of $T_{3}$. To find all the different kinds of t-o-m symmetry-breaking stretches, we must first list all the subgroups of 
$T_{3}$ in (2.2) that are themselves point groups of some basis. In $T_{3}$ there are the two following orthorhombic subgroups:

$$
\begin{aligned}
& O_{1,2,3}=\left\{\mathbf{1}, \boldsymbol{R}_{c_{1}}{ }^{\pi}, \boldsymbol{R}_{\boldsymbol{c}_{2}}{ }^{\pi}, \boldsymbol{R}_{\boldsymbol{c}_{3}}{ }^{\pi}\right\}, \\
& O_{3,1 \pm 2}=\left\{\mathbf{1}, \boldsymbol{R}_{\boldsymbol{c}_{3}}{ }^{\pi}, \boldsymbol{R}_{\boldsymbol{c}_{1}+\boldsymbol{c}_{2}}{ }^{\pi}, \boldsymbol{R}_{\boldsymbol{c}_{1}-\boldsymbol{c}_{2}}{ }^{\pi}\right\} .
\end{aligned}
$$

Furthermore, in $T_{3}$ there are five monoclinic subgroups:

$$
\begin{gathered}
M_{1}=\left\{\mathbf{1}, \boldsymbol{R}_{\boldsymbol{c}_{1}}{ }^{\pi}\right\}, M_{2}=\left\{\mathbf{1}, \boldsymbol{R}_{\boldsymbol{c}_{2}}{ }^{\pi}\right\}, \\
M_{3}=\left\{\mathbf{1}, \boldsymbol{R}_{\boldsymbol{c}_{3}}{ }^{\pi}\right\}, \\
M_{1+2}=\left\{\mathbf{1}, \boldsymbol{R}_{\boldsymbol{c}_{1}+\boldsymbol{c}_{2}}{ }^{\pi}\right\}, M_{1-2}=\left\{\mathbf{1}, \boldsymbol{R}_{\boldsymbol{c}_{1}-\boldsymbol{c}_{2}}{ }^{\pi}\right\} .
\end{gathered}
$$

Finally, in $T_{3}$ there is the trivial triclinic subgroup $\{\mathbf{1}\}$. The two monoclinic groups in (2.5) and (2.7) form in $T_{3}$ the conjugacy classes $M_{1,2}$ and $M_{1 \pm 2}$, respectively, while each one of the subgroups in (2.3), (2.4), and (2.6), forms a conjugacy class by itself. These inclusion and conjugacy relations are summarized in Fig. 3.

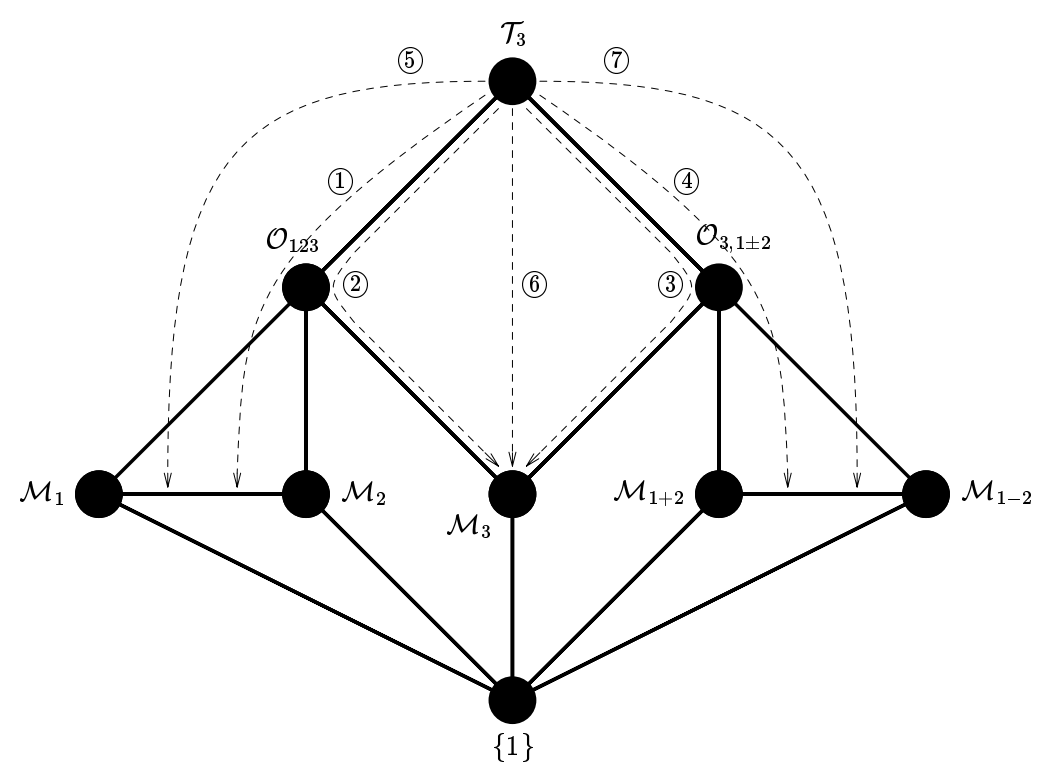

Fig. 3. Schematic representation of the point groups included in the tetragonal point group $T_{3}$. Indicated are the group-subgroup relations (slanted lines), and the conjugacy classes in $T_{3}$ (horizontal lines). The to-m symmetry-breaking paths, numbered as in the text, are indicated by the dotted lines.

If a symmetry-breaking stretch $\boldsymbol{U} \in N$ deforms the basis $\boldsymbol{t}_{\boldsymbol{a}}$ in a phase transition, also any other stretch of the form 
is as likely to break the symmetry of $\boldsymbol{t}_{a}$ in that phase change. To find all the distinct symmetry-breaking stretches that produce the subgroups indicated in (2.3)-(2.7) one must consider the orbits (2.8), also called 'variant structures'. The distinct stretches (2.8), which all belong to $N$, are called the 'variants' of $\boldsymbol{U}$. A standard argument (e.g. [PZ00]) shows that the number of variants is the ratio of the orders of the point groups of the parent and product phases. Thus, when $\boldsymbol{U}$ lowers the symmetry of $\boldsymbol{t}_{a}$ to orthorhombic there are two variants, while for a monoclinic low symmetry phase there are four variants. When different variant stretches $\boldsymbol{U}_{i}, i=1, \ldots n$, are applied to the basis $\boldsymbol{t}_{a}$, the point groups of the deformed bases $\boldsymbol{U}_{\mathrm{l}} \boldsymbol{t}_{a}, \ldots, \boldsymbol{U}_{n} \boldsymbol{t}_{a}$, do not in general coincide, nor are they all distinct; however, a simple check shows that they are all necessarily conjugate subgroups of $T_{3}$; that is why the conjugacy classes are indicated in Fig. 3. In Fig. 4 we give an explicit description of the variants for all the t-o-m symmetry-breaking stretches, together with the point groups of the deformed bases.

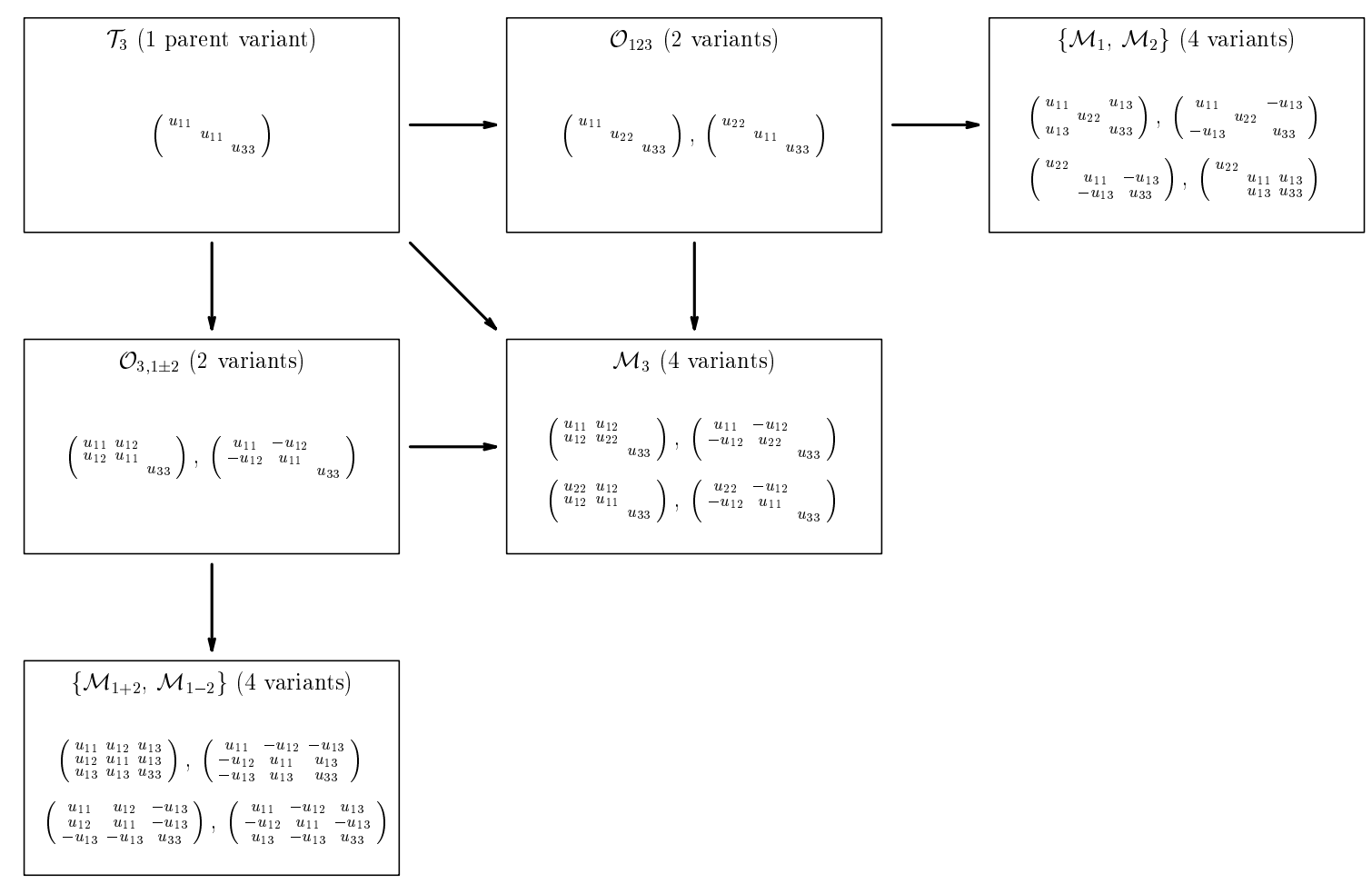

Fig. 4 The complete list of the t-o-m symmetry-breaking stretches. Indicated are also the point (sub)groups of $T_{3}$ that are obtained when such stretches are applied to the reference tetragonal basis $\boldsymbol{t}_{a}$.

Now, by inspection of Figs. 3-4 we determine all the kinematically distinct possibilities for lowering the symmetry of the parent lattice (2.1) from tetragonal, to orthorhombic, to monoclinic, or directly from tetragonal to monoclinic. At each step the 
symmetry of the reference basis $\boldsymbol{t}_{a}$ is lowered through the application of different sets of symmetry-breaking stretch variants as in Fig. 4, which produce distinct sets of lowersymmetry (orthorhombic or monoclinic) lattices with point groups as in (2.3)-(2.7).

There are two essentially distinct t-o transition paths, and four essentially distinct t-o$m$ paths $^{3}$ (a t-o path can be viewed as the first 'step' in a t-o-m path). Each path is indicated by the corresponding point groups of the variant structure produced by a deformation of the tetragonal basis $\boldsymbol{t}_{a}$. Each path 'starts' from the point group of $\boldsymbol{t}_{a}$, goes at first 'through' the point group of a set of two orthorhombic variants, and finally reaches the point group (s) indeed, the conjugacy class in $T_{3}$ - of a set of four monoclinic variants:

$$
\begin{aligned}
& \text { path (1) } \quad T_{3} \rightarrow O_{1,2,3} \rightarrow M_{1,2}, \quad \text { path (2) } \quad T_{3} \rightarrow O_{1,2,3} \rightarrow M_{3} \text {, } \\
& \text { path (3) } \quad T_{3} \rightarrow O_{3,1 \pm 2} \rightarrow M_{3}, \quad \text { path (4) } \quad T_{3} \rightarrow O_{3,1 \pm 2} \rightarrow M_{1 \pm 2} \text {. }
\end{aligned}
$$

One also has the following three direct $\mathrm{t}-\mathrm{m}$ transition paths:

$$
\text { path (5) } \quad T_{3} \rightarrow M_{1,2}, \quad \text { path (6) } \quad T_{3} \rightarrow M_{3}, \quad \text { path (7) } \quad T_{3} \rightarrow M_{1 \pm 2}
$$

(see Fig. 3). The paths above can all be completed with the final symmetry breaking to a set of 8 triclinic variants whose point group is the trivial subgroup $\{\mathbf{1}\}$. The direct tetragonal-totriclinic transition $T_{3} \rightarrow\{\mathbf{1}\}$ is also possible, but not relevant for our analysis.

We discuss below how some (although not all) of the above kinematic transition paths can be obtained through symmetry-breaking bifurcations originating from the (partial) softening of suitable moduli in the tetragonal and orthorhombic crystals.

\section{An example: zirconia}

As mentioned in the Introduction, the prototype for our general study of t-o-m crystals with a triple point is zirconia $\left(\mathrm{ZrO}_{2}\right)$, an important active material exhibiting tetragonal, orthorhombic and monoclinic phases. Detailed crystallographic descriptions of the zirconia polymorphs can be found in [KHH89, HKRH90, LTAP93, DL98, Kr98, FPF00]. We confine ourselves to temperatures and pressures that are not too high; the data in the literature indicate that the t-o-m polymorphs of $\mathrm{ZrO}_{2}$ can then be described by simple lattices whose unit cells contain a 'motif' with four variously arranged and deformed $\mathrm{Zr}-\mathrm{O}_{2}$ units. These lattices are all suitable deformations of the simple $\mathrm{Zr}$ lattice obtained by considering the sole corner atoms in the conventional f.c.c. cell of cubic $\mathrm{ZrO}_{2}$ (not taken into

3 The two t-o transformation mechanisms differ in how they break the symmetry of the primitive tetragonal basis $t_{a}$. The transition $T_{3} \rightarrow O_{1,2,3}$ breaks the equality condition for the lengths of the vectors $t_{1}$ and $t_{2}$, while maintaining them orthogonal. The transition $T_{3} \rightarrow O_{3,1 \pm 2}$, on the other hand, breaks the orthogonality of these two vectors, while maintaining them of the same length. The first transition produces a primitive orthorhombic lattice, while the second one produces a base-centered orthorhombic lattice. A similar analysis can be made for the four t-o-m transition paths (see [Z96, PZ00]). 
account here). Here we disregard the atoms of the motif, and concentrate only on the t-o-m deformations of this 'skeletal' lattice (see [ID89, FPF00] for a discussion of the motif deformations).

The structure giving the (most) stable phase of $\mathrm{ZrO}_{2}$ at temperatures around $1000^{\circ} \mathrm{C}$ and low pressures has a space group of the primitive tetragonal type (see for instance [LTAP93, FPF00]). The 'skeletal' primitive tetragonal cell that we consider as reference in (2.1) and Fig. 2 is obtained from the corner $\mathrm{Zr}$ atoms of the face-centered tetragonal cell of tetragonal zirconia.

The (most) stable phase of zirconia at the lower pressures and temperatures has a primitive monoclinic lattice. A summary of available experimental results regarding the $\mathrm{t}-\mathrm{m}$ orientation relationships in zirconia can be found in [FBV90, BFV91, Si97].

A third phase of zirconia, observed to be the most stable at lower temperatures and higher pressures, has orthorhombic symmetry. This phase is often referred to as 'orthoI' in the literature - see for instance [HKRH90, LTAP93, DL98]). No experimental study appears to indicate the orientation relationships for the tetragonal-to-orthoI transition. At higher pressures and temperatures zirconia also crystallizes in a number of other structures, which have been only partially investigated; [LTAP93] shows the complete phase diagram - see also [BJP85, KPA88, KHH89, HKRH90, OYKIN91, DL98, OM98]).

Figure 1 summarizes the above observations. In this portion of the phase diagram of $\mathrm{ZrO}_{2}$, the equilibrium phase boundaries are fairly well established, as is the location of the $\mathrm{t}$ $\mathrm{o}-\mathrm{m}$ triple point. The $\mathrm{o}-\mathrm{m}$ and $\mathrm{t}-\mathrm{m}$ lines have negative slopes and are almost parallel to each other; the t-o line has slightly positive slope and is almost parallel to the pressure axis. The observations also show hysteresis across the indicated phase boundaries. We take the diagram in Fig. 1 to be fairly typical of t-o-m crystals with a triple point; the goal of our study is to construct an energy function that is able to reproduce it.

When phrased in terms of the results of Section 2, the experimental observations on the t-o-m zirconia polymorphs that we are concerned with can be summarized as follows:

(A) The best-established $\mathrm{t}-\mathrm{m}$ orientation relationship for $\mathrm{ZrO}_{2}$ indicates that the fourfold axis of the tetragonal phase is parallel to the two-fold axis of the monoclinic phase (see [SMS74, BFV91]). This means that monoclinic $\mathrm{ZrO}_{2}$ comes from a variant structure with point group $M_{3}$. It should be mentioned that the orientation relationships compatible with the $M_{1,2}$-monoclinic variants have also been reported (see for instance [HM90, FBV90, Si97]).

(B) No data seem to indicate whether the orthoI phase of $\mathrm{ZrO}_{2}$ is $O_{1,2,3}$ or $O_{3,1 \pm 2}$. However, one can refer to the data on the positions of the two families of $\mathrm{O}$ atoms that, in the $\mathrm{ZrO}_{2}$ lattice, have coordination 3 and 4 with the $\mathrm{Zr}$ atoms (see [KHH89, HKRH90, LTAP93, DL98, FPF00]); the original configuration of these families in the tetragonal zirconia phase (in which all the $\mathrm{O}$ atoms have the same coordination 4) indicates that the orthoI phase of $\mathrm{ZrO}_{2}$ should derive from an $O_{1,2,3}$-orthorhombic variant structure (see also [HKRH90, Kr98]). The hypothesis of a $T_{3} \rightarrow O_{1,2,3}$ mechanism is also immediately consistent with the space group reported in the literature for the orthoI phase of zirconia. The associated crystallography needs some elaborate (although perhaps not impossible) motif adjustment to be made compatible with a $T_{3} \rightarrow O_{3,1 \pm 2}$ mechanism.

Following the above lines of indirect evidence, we proceed by assuming that the transformations associated with the triple point in t-o-m crystals are the following (see path (2) in Section 2): 
(i) the t-o transition is $T_{3} \rightarrow O_{1,2,3}$,

(ii) the o-m transition is $O_{1,2,3} \rightarrow M_{3}$,

(iii) the t-m transition is $T_{3} \rightarrow M_{3}$.

In the next Sections we develop a constitutive framework compatible with this kinematic picture and capable of describing both paths (2) and (3) of Section 2. Only at the end of Section 5, by making suitable assumptions concerning the order-parameter coupling and temperature dependence of the energy coefficients, we will select path (2). It is important to point out that in the previous literature the $\mathrm{t}-\mathrm{m}$ phase transformation in zirconia has been mostly regarded to be of a different nature than proposed here. Thus [Cha88, BT93, ST96], among others, advocated the direct path (5), leading to a model where a subcritical bifurcation associated with the softening of the tetragonal modulus $C_{44}$ drives the t-m phase transition ${ }^{4}$. However, as we have seen above, the experimental evidence rather suggests a $T_{3}$ $\rightarrow M_{3}$ mechanism. As we will see, the $T_{3} \rightarrow M_{3}$ path cannot be obtained through the softening of any tetragonal modulus. We thus envisage the $T_{3} \rightarrow M_{3}$ phase change as a firstorder transformation resulting from the coexistence of 'distant' energy wells with $T_{3}$ and $M_{3}$ symmetry; in this framework the monoclinic wells originate from $T_{3}$ through two successive subcritical bifurcations, involving also the $O_{1,2,3}$-orthorhombic phase (see Fig. 8).

\section{The energy function}

Consider a crystalline body in a configuration represented by a tetragonal lattice (2.1). For the phenomena we consider, it is safe to assume that the lattice deformation and the gross deformation of the crystal agree (this is the 'Cauchy-Born Rule', see for instance [Z96a]). We denote by $\boldsymbol{F}$ the deformation gradient of the macroscopic body, whose reference configuration we choose to coincide with the unstressed tetragonal configuration assumed by the crystal at any given temperature $T$; the temperature thus enters our discussion as a control parameter. As is usual in elasticity theory, we take the symmetric, positive-definite CauchyGreen tensor

$$
\boldsymbol{C}=\boldsymbol{F}^{t} \boldsymbol{F}=\boldsymbol{U}^{2}
$$

as a measure of the local strain in the crystal (here $\boldsymbol{U}$ is the stretch tensor appearing in the polar decomposition of $\boldsymbol{F}$ ). All the considerations made in Section 2 for $\boldsymbol{U}$ carry over to $\boldsymbol{C}$. The free energy per unit reference volume is assumed to be a function of $\boldsymbol{C}$ and of the temperature $T$ :

\footnotetext{
${ }^{4}$ We cannot completely exclude that this mechanism plays a role in the $\mathrm{t}-\mathrm{m}$ phase change of $\mathrm{ZrO}_{2}$, because the orientation relationships producing the $M_{1,2}$-monoclinic variants have indeed been reported in the literature.
} 


$$
\varphi=\hat{\varphi}(\boldsymbol{C}, T) .
$$

Here $\boldsymbol{C}$ varies in a neighborhood $N$ of the identity tensor $\mathbf{1}$, as mentioned in Section 2 (assumption of small but finite strains). We assume function $\varphi$ to have tetragonal invariance, that is:

$$
\varphi(\boldsymbol{C}, T)=\varphi\left(\boldsymbol{R}^{t} \boldsymbol{C R}, T\right)
$$

for all $T$, all $\boldsymbol{C}$, and all the rotations $\boldsymbol{R}$ in the group $T_{3}$ introduced in (2.2).

The six components of the Cauchy-Green tensor $c_{a b}=\boldsymbol{C} \boldsymbol{c}_{a} \cdot \boldsymbol{c}_{b}$ form a symmetric 3 by 3 matrix, which, following Voigt, is represented by a 6 -tuplet $\left(u_{1}, \ldots, u_{6}\right)$ :

$$
u_{1}=c_{11}, \quad u_{2}=c_{22}, \quad u_{3}=c_{33}, \quad u_{4}=c_{23}, \quad u_{5}=c_{13}, \quad u_{6}=c_{12} .
$$

The variables $u_{A}, A=1, \ldots, 6$, are the coefficients in the representation of the tensor $C$ as a linear combination of the (orthonormal) tensor basis $\boldsymbol{V}_{1}, \boldsymbol{V}_{2}, \ldots, \boldsymbol{V}_{6}$ :

$$
C=\sum u_{A} \mathbf{V}_{A},
$$

where

$$
\boldsymbol{V}_{1}=\boldsymbol{c}_{1} \otimes \boldsymbol{c}_{1}=\left(\begin{array}{ccc}
1 & 0 & 0 \\
0 & 0 & 0 \\
0 & 0 & 0
\end{array}\right), \quad \ldots, \quad \boldsymbol{V}_{4}=\boldsymbol{c}_{2} \otimes \boldsymbol{c}_{3}+\boldsymbol{c}_{3} \otimes \boldsymbol{c}_{2}=\left(\begin{array}{ccc}
0 & 0 & 0 \\
0 & 0 & 1 \\
0 & 1 & 0
\end{array}\right), \ldots
$$

In terms of the six variables $u_{A}$ the free energy in (4.2) writes

$$
\varphi=\bar{\varphi}\left(u_{A}, T\right) .
$$

We introduce the strain tensor $\boldsymbol{E}$ defined by

$$
\boldsymbol{E}=\frac{1}{2}(\boldsymbol{C}-\mathbf{1})=\sum e_{A} \mathbf{V}_{A},
$$

with the corresponding Voigt 6-tuplet $e_{A}$. The problem now consists in constructing the most general low-order polynomial expansion of $\bar{\varphi}$ in terms of the variables $e_{A}$, compatible with the symmetry (4.3) and reproducing the phase diagram in Fig. 1.

Notice that the zero-order term in this expansion depends only on temperature, and for our purposes can be neglected. Furthermore, since the reference configuration is an equilibrium one at temperature $T$, the first-order terms are also absent from the free energy expansion. We thus begin with the quadratic term

$$
\bar{\varphi}_{Q}=\frac{1}{2} C_{A B} e_{A} e_{B},
$$


where, due to the tetragonal symmetry, in our basis $\boldsymbol{c}_{a}$ we have ([Lo27, LL86]):

$$
C_{A B}=C_{B A}=\left(\begin{array}{llllll}
C_{11} & C_{12} & C_{13} & & & \\
& C_{11} & C_{13} & & & \\
& & C_{33} & & & \\
& & & C_{44} & & \\
& & & & C_{44} & \\
& & & & & C_{66}
\end{array}\right) .
$$

The six material parameters $C_{11}, C_{12}, C_{13}, C_{33}, C_{44}, C_{66}$, completely characterize the tetragonal crystal in the limit of linear elasticity, and, in general, are temperature dependent. As usual, $C_{A B}$ in (4.10) can be regarded as the matrix representation of a symmetric linear operator with respect to the standard tensor basis $\boldsymbol{V}_{A}$ in (4.6). A straightforward calculation shows that for a generic case $C_{13} \neq 0$, the (mutually orthogonal) eigen-spaces and the corresponding eigen-values of this operator are as follows:

Eigen-space

$$
\begin{aligned}
& B_{1}=\left\langle V_{1}+V_{2}+\beta_{1} V_{3}\right\rangle \\
& B_{2}=\left\langle V_{1}+V_{2}+\beta_{2} V_{3}\right\rangle \\
& B_{3}=\left\langle V_{1}-V_{2}\right\rangle \\
& B_{4}=\left\langle V_{4}, V_{5}\right\rangle \\
& B_{5}=\left\langle V_{6}\right\rangle
\end{aligned}
$$

Eigen-value (elastic modulus)

$$
\begin{aligned}
& \lambda_{1} \\
& \lambda_{2} \\
& C_{11}-C_{12} \\
& C_{44} \text { (double) } \\
& C_{66},
\end{aligned}
$$

where the four numbers $\beta_{1}, \beta_{2}, \lambda_{1}$ and $\lambda_{2}$ are the roots of the following quadratic equations

$$
\beta^{2}+\left(C_{11}+C_{12}-C_{33}\right) C_{13}^{-1} \beta-2=0
$$

$$
\lambda^{2}-\left(C_{11}+C_{12}+C_{33}\right) \lambda+\left(C_{11}+C_{12}\right) C_{33}-2 C_{13}^{2}=0
$$

From the orthogonal decomposition in the eigen-spaces $B_{1}, \ldots B_{5}$, one can obtain a basis of eigen-strains which diagonalizes the matrix of the elastic constants $C_{A B}$. However, for the 
purpose of treating hydrostatic loads, we introduce an alternative (orthogonal) tensor basis $\boldsymbol{W}_{A}$, as follows

$$
\begin{array}{r}
\boldsymbol{W}_{1}=\frac{1}{3}\left(\boldsymbol{V}_{1}+\boldsymbol{V}_{2}+\boldsymbol{V}_{3}\right)=\frac{1}{3} \mathbf{1}, \quad \boldsymbol{W}_{2}=\boldsymbol{V}_{1}+\boldsymbol{V}_{2}-2 \boldsymbol{V}_{3}, \\
\boldsymbol{W}_{3}=\frac{1}{\sqrt{2}}\left(\boldsymbol{V}_{1}-\boldsymbol{V}_{2}\right), \quad \boldsymbol{W}_{r}=\boldsymbol{V}_{r}, \quad r=4,5,6 .
\end{array}
$$

The new strains $\boldsymbol{W}_{3}, \ldots, \boldsymbol{W}_{6}$, are eigen-strains of the elasticity tensor, so that the basis $\boldsymbol{W}_{A}$ diagonalizes $C_{A B}$ except for the first two directions $\boldsymbol{W}_{1}$ and $\boldsymbol{W}_{2}$. By writing

$$
\boldsymbol{E}=\sum y_{A} \boldsymbol{W}_{A}
$$

we obtain a new set of strain coordinates $y_{A}$, whose relation to the original coordinates $e_{A}$ is given by

$$
y_{1}=e_{1}+e_{2}+e_{3}, \quad y_{2}=\frac{1}{6}\left(e_{1}+e_{2}-2 e_{3}\right), \quad y_{3}=\frac{1}{\sqrt{2}}\left(e_{1}-e_{2}\right)
$$

$$
y_{4}=e_{4}, \quad y_{5}=e_{5}, \quad y_{6}=e_{6} .
$$

In particular, our new parameter $y_{1}$ characterizes dilatation in the linear approximation; the potential energy of the hydrostatic pressure $p$ can then be approximated by the term $p y_{1}$.

As mentioned above, the new variables $y_{A}$ almost diagonalize the quadratic part of the energy, giving

$$
\bar{\varphi}_{Q}=\frac{1}{2} \bar{C}_{A B} y_{A} y_{B},
$$

with

$$
\bar{C}_{A B}=\bar{C}_{B A}=\left(\begin{array}{llllll}
A_{11} & A_{12} & & & & \\
& A_{22} & & & & \\
& & C_{11}-C_{12} & & & \\
& & & C_{44} & & \\
& & & C_{44} & \\
& & & & & C_{66}
\end{array}\right) \text {, }
$$

where the new elastic moduli $A_{11}, A_{12}$ and $A_{22}$ are given by

$$
A_{11}=\frac{1}{9}\left[2\left(C_{11}+C_{12}\right)+4 C_{13}+C_{33}\right]
$$




$$
\begin{aligned}
& A_{12}=\frac{2}{3}\left[C_{11}+C_{12}-C_{13}-C_{33}\right], \\
& A_{22}=2\left[C_{11}+C_{12}-4 C_{13}+2 C_{33}\right] .
\end{aligned}
$$

The quadratic part (4.15) of the energy thus writes explicitly:

$$
\bar{\varphi}_{Q}=\frac{1}{2}\left[A_{11} y_{1}^{2}+2 A_{12} y_{1} y_{2}+A_{22} y_{2}^{2}+\left(C_{11}-C_{12}\right) y_{3}^{2}+C_{44}\left(y_{4}^{2}+y_{5}^{2}\right)+C_{66} y_{6}^{2}\right] .
$$

We can now clarify the kinematical nature of the variables $y_{A}$ introduced above, and specify the relevant order parameters (see also [Cha88]). First, notice that the application of any linear combination of the strains $\boldsymbol{W}_{1}$ and $\boldsymbol{W}_{2}$, does not change the $T_{3}$-symmetry of the reference basis. The variables $y_{1}$ and $y_{2}$ are therefore ' $T_{3}$-symmetry-preserving'. During the $\mathrm{t}-\mathrm{o}-\mathrm{m}$ transition processes these variables follow the evolution of the order parameters and adjust as necessary to secure equilibrium. Since we are not interested in the modulus softening associated with any tetragonal-to-tetragonal transformation, we assume that the matrix $A_{I J}(I, J=1,2)$ in (4.16) is positive-definite.

Next, we observe that any generic combination of the eigen-strains $\boldsymbol{W}_{4}$ and $\boldsymbol{W}_{5}$ produces a triclinic basis with a trivial point group. However, application of either $\boldsymbol{W}_{4}$ or $\boldsymbol{W}_{5}$ alone produces a $T_{3} \rightarrow M_{1,2}$ symmetry breaking, while any of the strains $\boldsymbol{W}_{4} \pm \boldsymbol{W}_{5}$ produce a transition $T_{3} \rightarrow M_{1 \pm 2}$. We conclude that these two t-m transitions are linked to the strain variables $y_{4}$ and $y_{5}$, and are caused by the softening of the modulus $C_{44}$ (see [E93, PZ00] for the analysis of the corresponding bifurcations, and [BT93, ST96] for a study of the global picture). Since we exclude both of these t-m paths, we assume the modulus $C_{44}$ to be positive; notice that our theory must also be compatible with the fact that the value of the strain variables $y_{4}$ and $y_{5}$ must indeed be zero along the chosen t-o-m paths (2) and (3).

Finally, the application of a combination of the eigen-strains $y_{3} \boldsymbol{W}_{3}+y_{6} \boldsymbol{W}_{6}$ to the reference tetragonal basis produces: (i) a basis with $O_{1,2,3}$-symmetry, if $y_{3} \neq 0$ and $y_{6}=0$; (ii) a basis with $O_{3.1 \pm 2}$-symmetry if $y_{3}=0$ and $y_{6} \neq 0$; and (iii) a basis with $M_{3}$-symmetry if both $y_{3}$ and $y_{6}$ are different from zero. From the form of the elasticity matrix $\bar{C}_{A B}$ in (4.16), we conclude that a softening of the modulus $C_{66}$ activates the variable $y_{6}$ and produces a $T_{3}$ $\rightarrow O_{3,1 \pm 2}$ bifurcation, while a softening of the modulus $C_{11}-C_{12}$ activates $y_{3}$ and produces a $T_{3} \rightarrow O_{1,2,3}$ bifurcation. The relevant t-o-m transition paths (2) and (3) are thus described by the order parameters $y_{3}$ and $y_{6}$, and our model is essentially two-dimensional.

The non-quadratic part of the free energy density, respecting the symmetry (4.3), can be expressed as a function of suitable polynomial invariants of the tetragonal group, whose complete list can be found in [SM58]. In the spirit of Landau theory, we seek the lowestorder expression for the energy function, with the minimal coupling between the order 
parameters and between the latter and the rest of the strain components. The order of the polynomial depends on the number of symmetry-related energy wells that must be considered. In this case we need terms of at least sixth order in $y_{3}$ and $y_{6}$; the corresponding non-quadratic tetragonal invariants are:

$$
y_{3}^{4}, \quad y_{6}^{4}, \quad y_{3}^{2} y_{6}^{2}, \quad y_{3}^{6}, \quad y_{6}^{6}, \quad y_{3}{ }^{4} y_{6}^{2}, \quad y_{3}{ }^{2} y_{6}{ }^{4} \text {. }
$$

We keep the first five terms in this list and omit the last two, as the bi-quadratic term $y_{3}^{2} y_{6}^{2}$ already gives enough order-parameter coupling. Furthermore, following [SD86, BT93, ST96], and in order to produce a triggering mechanism that allows the hydrostatic pressure $p$ to influence the behavior of the shear-related order parameters, we consider the following third-degree tetragonal invariants that couple the dilatation $y_{1}$ with the shears $y_{3}$ and $y_{6}$ :

$$
y_{1} y_{3}^{2}, \quad y_{1} y_{6}^{2}
$$

We can now write explicitly our 'minimal' proposal for the non-quadratic part of the free energy density 5 :

$$
\bar{\varphi}_{N Q}=\frac{1}{2} H_{3} y_{1} y_{3}{ }^{2}+\frac{1}{2} H_{6} y_{1} y_{6}{ }^{2}+\frac{1}{4} D_{3} y_{3}{ }^{4}+\frac{1}{4} D_{6} y_{6}{ }^{4}+\frac{1}{2} L y_{3}{ }^{2} y_{6}{ }^{2}+\frac{1}{6} K_{3} y_{3}{ }^{6}+\frac{1}{6} K_{6} y_{6}{ }^{6} .
$$

By combining (4.18) and (4.21) with the potential of the loading device we obtain the final expression for the Gibbs energy density:

$$
g=\varphi_{0}(T)+\bar{\varphi}_{Q}\left(y_{A}, T\right)+\bar{\varphi}_{N Q}\left(y_{A}, T\right)+p y_{1} .
$$

Here, $\varphi_{0}(T)$ is the energy of the temperature-dependent tetragonal reference state, whose specific form is not important for the purpose of this study. We remark that the energy (4.21) belongs to the general class of energies analyzed in [SD86]; due to the loading and the presence of the triple point, our conclusions will considerably differ from theirs.

\section{Equilibria and phase diagram}

With the energy expression given by (4.22), the homogeneous equilibrium configurations of the t-o-m crystal (critical points of $g$ at fixed $p$ and $T$ ) can be found from the equations (recall (4.17)):

\footnotetext{
5 We stress that our present discussion of a t-o-m constitutive framework is 'minimal', as some of the effects that are not relevant for the existence of a triple point are purposefully neglected. Thus, with the energy as in (4.21), the anisotropy of both the orthorhombic and monoclinic low-symmetry phases is incomplete; in (4.21) one should add, at least, the coupling terms $e_{4} e_{5} e_{6}$ and $e_{1} e_{4}{ }^{2}+e_{2} e_{5}^{2}$, that is, $y_{4} y_{5} y_{6}, y_{1}($ $\left.y_{4}{ }^{2}+y_{5}{ }^{2}\right), y_{2}\left(y_{4}{ }^{2}+y_{5}{ }^{2}\right)$, and $y_{3}\left(y_{4}{ }^{2}-y_{5}{ }^{2}\right)-$ see (4.8) and (4.14). The latter are necessary to obtain the correct symmetries in the elastic moduli of the orthorhombic and monoclinic phases.
} 


$$
\begin{aligned}
& A_{11} y_{1}+A_{12} y_{2}+\frac{1}{2} H_{6} y_{6}^{2}+\frac{1}{2} H_{3} y_{3}^{2}+p=0, \\
& A_{12} y_{1}+A_{22} y_{2}=0, \\
& \left(C_{11}-C_{12}\right) y_{3}+H_{3} y_{1} y_{3}+D_{3} y_{3}^{3}+K_{3} y_{3}^{5}+L y_{3} y_{6}{ }^{2}=0, \\
& C_{66} y_{6}+H_{6} y_{1} y_{6}+D_{6} y_{6}{ }^{3}+K_{6} y_{6}^{5}+L y_{6} y_{3}^{2}=0, \\
& C_{44} y_{4}=0 \\
& C_{44} y_{5}=0
\end{aligned}
$$

This system can be solved for the non-order-parameter strain variables $y_{1}, y_{2}, y_{4}, y_{5}$, which enter the energy at most quadratically. We obtain:

$$
\begin{gathered}
y_{1}=-\frac{1}{2 M}\left(H_{3} y_{3}{ }^{2}+H_{6} y_{6}{ }^{2}+2 p\right) \\
y_{2}=-\frac{A_{12}}{A_{22}} y_{1}, \\
y_{4}=0, \quad y_{5}=0,
\end{gathered}
$$

where

$$
M=A_{11}-A_{12}^{2} / A_{22}>0
$$

is the effective bulk modulus of the tetragonal phase, which is positive due to the positivedefiniteness of the matrix $A_{I J}$ in (4.17).

When the order parameters $y_{3}$ and $y_{6}$ vanish, the first two equations in (5.2) describe the anisotropic compressibility of the parent tetragonal phase at temperature $T$ and pressure $p$. For non-vanishing $y_{3}$ or $y_{6}$, the same equations describe how the 'tetragonal' variables $y_{1}$ and $y_{2}$ adjust in the orthorhombic and monoclinic phases. The trivial solutions for $y_{4}$ and $y_{5}$ express the fact that no equilibria in this model have monoclinic point groups of the type (2.5) or (2.7).

If we now substitute into the Gibbs energy (4.22) the expressions for the equilibrium values of the non-order-parameter strain variables (5.2), and drop the terms that depend only on pressure and temperature, we obtain the Landau energy

$$
g_{L}=\frac{1}{2} G_{3} y_{3}{ }^{2}+\frac{1}{2} G_{6} y_{6}{ }^{2}+\frac{1}{4} \tilde{D}_{3} y_{3}{ }^{4}+\frac{1}{4} \tilde{D}_{6} y_{6}{ }^{4}+\frac{1}{2} \tilde{L} y_{3}{ }^{2} y_{6}{ }^{2}+\frac{1}{6} K_{3} y_{3}{ }^{6}+\frac{1}{6} K_{6} y_{6}{ }^{6},
$$

where we introduced the renormalized Landau coefficients: 


$$
\begin{gathered}
\tilde{L}=L-\frac{H_{3} H_{6}}{2 M}, \\
G_{3}=C_{11}-C_{12}-\frac{H_{3}}{M} p, \quad G_{6}=C_{66}-\frac{H_{6}}{M} p, \\
\tilde{D}_{3}=D_{3}-\frac{H_{3}{ }^{2}}{2 M}, \quad \tilde{D}_{6}=D_{6}-\frac{H_{6}{ }^{2}}{2 M} .
\end{gathered}
$$

In terms of the new coefficients, the equilibrium conditions for $y_{3}$ and $y_{6}$ take the form

$$
y_{3}\left(G_{3}+\tilde{L} y_{6}{ }^{2}+\tilde{D}_{3} y_{3}{ }^{2}+K_{3} y_{3}{ }^{4}\right)=0, \quad y_{6}\left(G_{6}+\tilde{L} y_{3}{ }^{2}+\tilde{D}_{6} y_{6}{ }^{2}+K_{6} y_{6}{ }^{4}\right)=0,
$$

Equations (5.5) describe the families of critical points for the Landau energy, parametrized by $p$ and $T$. The local minima of the energy (metastable configurations) are selected by the additional condition that the Hessian matrix $\partial^{2} g_{L} / \partial y_{3} \partial y_{6}$ is positive-definite. By using (5.3) we can write the corresponding inequalities explicitly

$$
\begin{gathered}
G_{6}+\tilde{L} y_{3}{ }^{2}+3 \tilde{D}_{6} y_{6}{ }^{2}+5 K_{6} y_{6}{ }^{4} \geq 0 \\
\left(G_{6}+\tilde{L} y_{3}{ }^{2}+3 \tilde{D}_{6} y_{6}{ }^{2}+5 K_{6} y_{6}{ }^{4}\right)\left(G_{3}+\tilde{L} y_{6}{ }^{2}+3 \tilde{D}_{3} y_{3}{ }^{2}+5 K_{3} y_{3}{ }^{4}\right) \geq 4 \tilde{L}^{2} y_{3}{ }^{2} y_{6}{ }^{2} .
\end{gathered}
$$

The Hessian determinant vanishes when $(5.6)_{2}$ holds as an equality: this condition specifies the stability boundaries of the various phases in the $(T, p)$-space, and provides the locations for the bifurcation points where distinct branches of extremals intersect.

Since now we are only considering the two-dimensional order-parameter space, and since the Landau energy function is even in $y_{3}, y_{6}$, we introduce the new variables

$$
\varepsilon=y_{3}^{2}, \quad \delta=y_{6}^{2},
$$

and, for uniformity, re-label the coefficients as follows:

$$
G_{\varepsilon} \equiv G_{3}, \quad G_{\delta} \equiv G_{6}, \quad D_{\varepsilon} \equiv \tilde{D}_{3}, \quad D_{\delta} \equiv \tilde{D}_{6}, \quad K_{\varepsilon} \equiv K_{3}, \quad K_{\delta} \equiv K_{6} .
$$

The solutions of the system (5.5), (5.7), are located in the first quadrant of the $(\varepsilon, \delta)$-plane, and have a transparent geometrical interpretation. The intersection of the coordinate axes

$$
\varepsilon=0 \quad \text { and } \quad \delta=0
$$


in the $(\varepsilon, \delta)$-plane gives the $T_{3}$-tetragonal (parent) phase, which is always a solution of the equilibrium equations. Then, any intersections of one of the parabolae

$$
G_{\delta}+\tilde{L} \varepsilon+D_{\delta} \delta+K_{\delta} \delta^{2}=0, \quad G_{\varepsilon}+\tilde{L} \delta+D_{\varepsilon} \varepsilon+K_{\varepsilon} \varepsilon^{2}=0
$$

with the coordinate axis orthogonal to its own axis gives orthorhombic equilibria which are either $O_{3,1 \pm 2}$ or $O_{1,2,3}$, depending respectively on whether $\varepsilon$ or $\delta$ vanish. Finally, any intersections of the two parabolae (5.8) with each other provide critical points with non-zero $\varepsilon$ and $\delta$, which give $M_{3}$-monoclinic equilibria (see Fig. 5). All these critical points ${ }^{6}$ are (meta)stable or unstable depending on whether conditions (5.6) are satisfied, which in turn depends on the values of the energy coefficients. Below, we discuss a choice of coefficients that assures the existence of a t-o-m triple point with the properties discussed in Section 3.

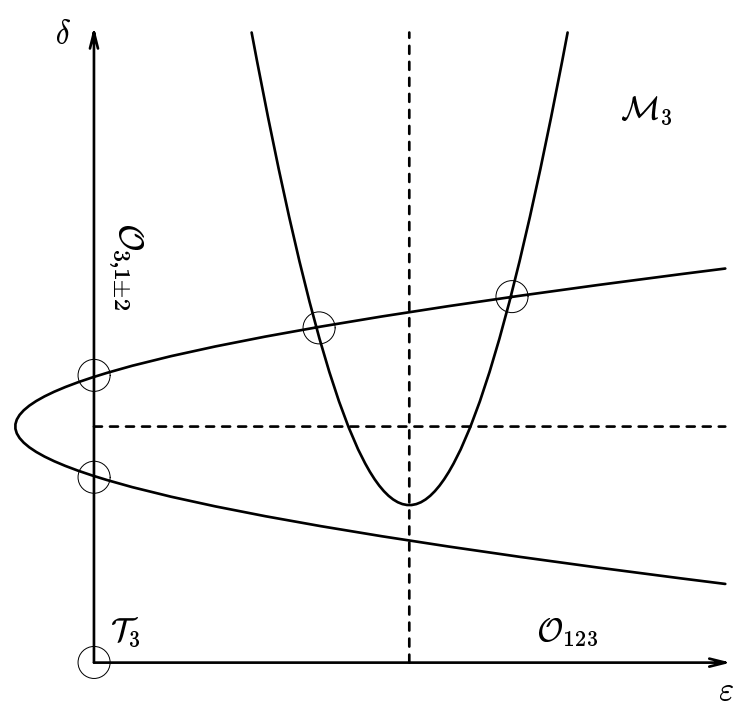

Fig. 5. The $(\varepsilon, \delta)$-plane, where the t-o-m phases are located as follows: $T_{3}$ is at the origin; $O_{3,1 \pm 2}$ and $O_{1,2,3}$ are on the $\delta$-and $\varepsilon$-axes, respectively; $M_{3}$ is in the interior of the first quadrant. Also shown are the two parabolae (5.10) whose intersection points between each other and with the coordinate axes (circled points) give the solutions of the equilibrium equations (5.5), (5.7).

First, for growth (and existence) reasons we assume that

$$
K_{\varepsilon}>0, \quad K_{\delta}>0 .
$$

As we have seen, the trivial solution $\varepsilon=0, \delta=0$, always exists, providing the $T_{3}$-parent phase for all $T$ and $p$. According to (5.6) this solution is (meta)stable if

$$
G_{\varepsilon}>0, \quad G_{\delta}>0 .
$$

6 We recall that our Landau energy (5.3) depends only on the squares $(\varepsilon, \delta)$ of the order parameters $\left(y_{3}, y_{6}\right)$, so that each stable root $(\varepsilon, \delta)$ of the equilibrium equations (5.5-7) actually gives a number of energy wells in the $\left(y_{3}, y_{6}\right)$-plane that describe a set of symmetry-related phase variants. 
When one of these $(p, T)$-dependent moduli passes through zero, the parent phase becomes unstable. The corresponding lines on the $p-T$ phase diagram mark the bifurcation points where the tetragonal equilibrium branch is connected with one of the orthorhombic branches characterized by either $\varepsilon \neq 0$ or $\delta \neq 0$. Specifically, the equilibria with $O_{1,2,3}$-symmetry are the non-trivial solutions of (5.5) with $\delta=0$ and non-zero $\varepsilon$, which correspond to the intersection of the parabola $(5.8)_{1}$ with the axis $\delta=0$. The values of the order parameters along this branch are given by:

$$
\delta=0, \quad \varepsilon=\frac{-D_{\varepsilon} \pm \sqrt{D_{\varepsilon}{ }^{2}-4 K_{\varepsilon} G_{\varepsilon}}}{2 K_{\varepsilon}} .
$$

The corresponding orthorhombic energy wells exist in the range of parameters defined by

$$
G_{\varepsilon} \leq \frac{D_{\varepsilon}^{2}}{4 K_{\varepsilon}}
$$

From (5.12-13) one can see that when the coefficient of the forth-order term

$$
D_{\varepsilon}<0
$$

there exists a domain of coexistence for the tetragonal and orthorhombic solutions. Notice that (5.15) will also make bifurcation along parameterized path in the $p-T$ plane subcritical. The line in the $p-T$ coordinates where (5.14) holds as an equality corresponds to turning points on the orthorhombic branch. Since in our system there is experimental evidence of hysteresis, we assume (5.15) to hold, indicating that the t-o transformation is of the first order.

The second bifurcation, from the orthorhombic phase $O_{1,2,3}$ to the monoclinic phase $M_{3}$, takes place as the Hessian matrix degenerates once again, when

$$
G_{\delta}+\tilde{L} \varepsilon=0
$$

Here $\varepsilon$ satisfies $(5.13)_{2}$. The corresponding boundary in the space of parameters is given by

$$
G_{\varepsilon}=\frac{G_{\delta}}{\tilde{L}}\left(D_{\varepsilon}-\frac{K_{\varepsilon} G_{\delta}}{\widetilde{L}}\right)
$$

A straightforward analysis shows that a domain of coexistence for o-m phases exists when

$$
D_{\delta}<0
$$

Since there is experimental evidence of hysteresis in the o-m phase change, we assume also (5.18) to hold (first-order o-m transformation). 
Due to the symmetry between the two order parameters in the Landau energy (5.3), the solutions with $O_{3,1 \pm 2}$ symmetry and their bifurcations can be discussed along the same lines. The corresponding critical points and their existence domain are given by formulas analogous to the above ones; in particular, conditions (5.15) and (5.18) imply that both the bifurcations $T_{3} \rightarrow O_{3,1 \pm 2}$ and $O_{3,1 \pm 2} \rightarrow M_{3}$ are subcritical.

The $M_{3}$-monoclinic equilibria of the system correspond to the solutions of (5.9) with $\varepsilon$ and $\delta$ different from zero. In the $(\varepsilon, \delta)$-plane the two parabolae in (5.9) may have up to four intersections (see Fig. 5). Their existence range can be found by imposing the condition that the two parabolae touch at one point and have no other intersections; this means that both (5.10) and (5.6) 2 taken as an equality hold simultaneously. Specifically, we obtain an overdetermined system of three equations

$$
\begin{gathered}
G_{\varepsilon}+\tilde{L} \delta+D_{\varepsilon} \varepsilon+K_{\varepsilon} \varepsilon^{2}=0, \quad G_{\delta}+\tilde{L} \varepsilon+D_{\delta} \delta+K_{\delta} \delta^{2}=0 \\
\left(G_{6}+\tilde{L} \varepsilon+3 \tilde{D}_{6} \delta+5 K_{6} \delta^{2}\right)\left(G_{3}+\tilde{L} \delta+3 \tilde{D}_{3} \varepsilon+5 K_{3} \varepsilon^{2}\right)=4 \tilde{L}^{2} \varepsilon \delta
\end{gathered}
$$

for two unknowns $\varepsilon$ and $\delta$. The resulting restriction on the coefficients of the Landau energy specifies the boundary of the existence domain for the phase $M_{3}$ in the space of parameters.

We now briefly discuss the stability conditions for the energy extremals described above. As already mentioned, the parent tetragonal phase $T_{3}$ is stable when conditions (5.12) hold. To find where the $O_{1,2,3}$ equilibria (5.13) are stable (their existence domain is given by (5.14)), we apply the inequalities (5.6), obtaining

$$
\begin{gathered}
G_{\varepsilon}+3 D_{\varepsilon} \varepsilon+5 K_{\varepsilon} \varepsilon^{2} \geq 0, \\
G_{\delta}+\tilde{L} \varepsilon \geq 0,
\end{gathered}
$$

with $\varepsilon$ again given by (5.13) 2 . The first of the stability conditions (5.20) does not restrict the coefficients of the Landau energy, but selects as the only stable root the one with the plus sign in $(5.13)_{2}$. The second condition in (5.20), taken as an equality, defines a line of bifurcation points where the $O_{1,2,3}$-branch with $\delta=0$ meets the $M_{3}$-branch with both $\varepsilon$ and $\delta$ different from zero - see (5.16-17). Now, before making the stability range of the orthorhombic phase $O_{1,2,3}$ explicit, we make the following digression.

In order to have the t-o-m triple point on the phase diagram, we need a domain in which all three stable phases, $T_{3}, O_{1,2,3}$ and $M_{3}$, coexist. It can be checked that the necessary and sufficient conditions for this to happen are found by imposing the existence region (5.12) for the $M_{3}$-minimizers defined by (5.19) to overlap with the stability region of the $T_{3^{-}}$ minimizer. The resulting equations are not very explicit; however, a sufficient condition is easy to obtain. Indeed, by definition $\varepsilon \geq 0$ when the (metastable) orthorhombic branch (5.13) exists; furthermore, because of (5.12), we have $G_{\delta} \geq 0$ on the stable part of the tetragonal branch. Then the condition 


$$
\tilde{L} \leq 0
$$

guarantees that the orthorhombic elastic modulus $G_{\delta}+N \mathcal{E}$ in $(5.20)_{2}$ can be zero within the stability limits of the tetragonal phase. This, together with the fact that both the t-o and the o$\mathrm{m}$ bifurcations are subcritical due to (5.15) and (5.18), guarantees that the monoclinic equilibria are stable in a domain where both the two other phases, tetragonal and orthorhombic, are stable, and thus the possibility that a t-o-m triple point exists.

Granted (5.21), we see from (5.13) and (5.20) that the $O_{1,2,3}$ phase is stable in the domain given by

$$
G_{\varepsilon} \geq \frac{G_{\delta}}{\tilde{L}}\left(D_{\varepsilon}-\frac{K_{\varepsilon} G_{\delta}}{\widetilde{L}}\right), \quad G_{\delta} \geq \frac{\tilde{L} D_{\varepsilon}}{2 K_{\varepsilon}}
$$

By combining (5.14) and (5.22) we obtain a complete description of the stability range for the orthorhombic phase $O_{1,2,3}$. The procedure to determine the stability range of the orthorhombic phase $O_{3,1 \pm 2}$ is completely analogous.

As we have already seen, the monoclinic equilibria $M_{3}$ originate at the o-m bifurcation points (5.17). Within the existence domain of the $M_{3}$ phase limited by (5.19), the stable roots can be selected by applying both inequalties (5.6); the resulting conditions in general are not explicit; we consider an example below.

In order to determine explicitly the structure of the $(p, T)$ phase diagram, we begin, as usual, with an analysis of the diagram in the space of the coefficients $G_{\varepsilon}$ and $G_{\delta}$. We first fix the values of the other Landau coefficients, respecting the sign conditions obtained above. By suitably rescaling the order parameters we can choose the coefficients $D_{\varepsilon}$ and $D_{\delta}$ to be equal to -1 . Then, to consider a simplified (and symmetric) case, we take $K_{\varepsilon}=K_{\delta}=1$, and $\tilde{L}=-1$. The resulting t-o-m phase diagram in the $\left(G_{\varepsilon}, G_{\delta}\right)$-plane is presented in Fig. 6. 


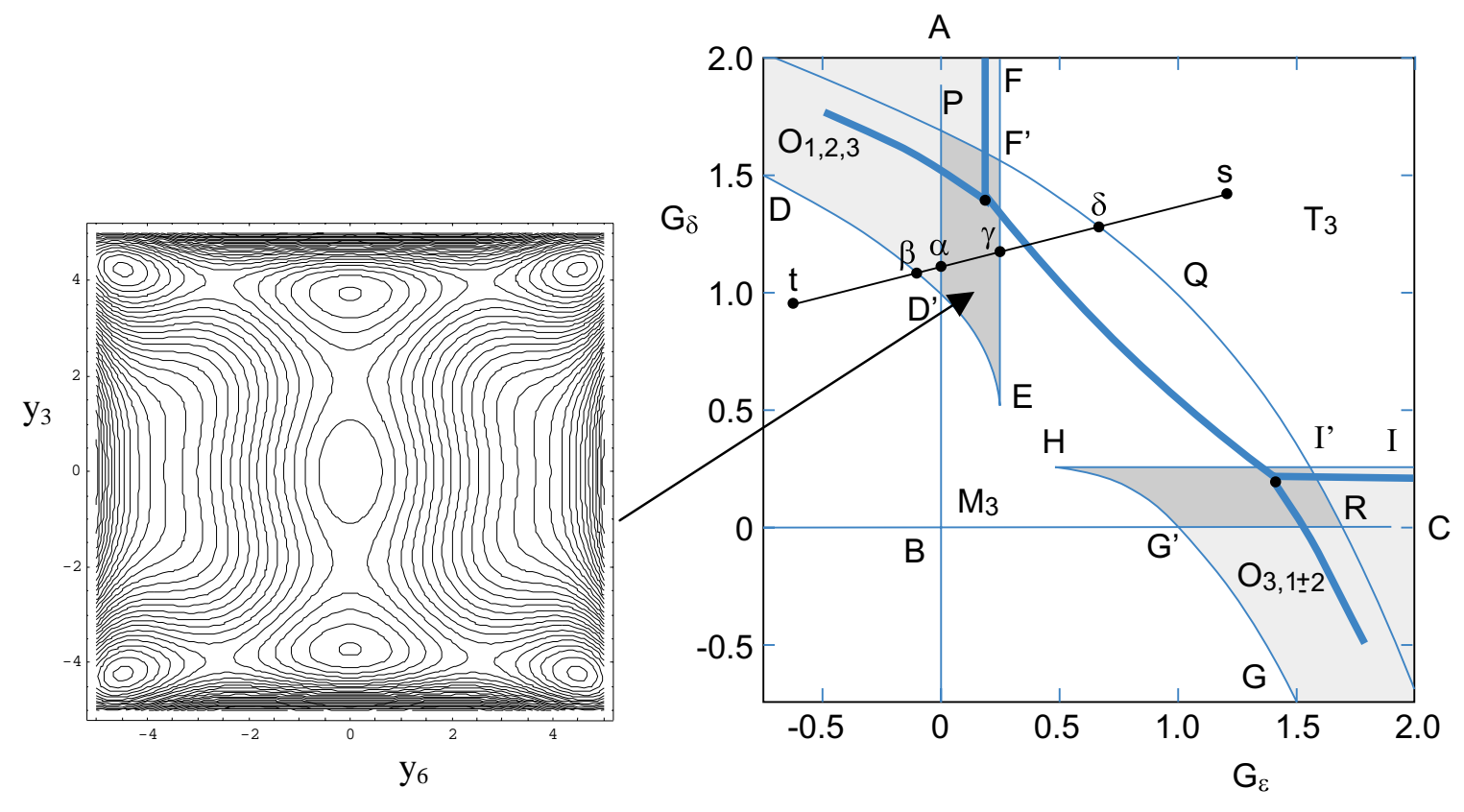

Fig. 6. Phase diagram in the $\left(G_{\mathcal{E}}, G_{\delta}\right)$-plane, obtained numerically from the Landau free energy (5.3) for coefficients as discussed in Section 5. Indicated are the (co)existence regions for the various phases described in the text, and the corresponding Maxwell lines (thick lines). The insert shows the level plots of a representative Landau energy landscape in the area $P D^{\prime} E F^{\prime}$ where the phases with $T_{3}, O_{1,2,3}$, and $M_{3}$ symmetries are all (meta)stable, showing a total of seven energy wells. The bifurcation diagram along the $t-s$ section is shown in Fig. 8.

On the $\left(G_{\varepsilon}, G_{\delta}\right)$-plane we can locate the (meta)stability domains for one, two, or three distinct sets of energy extremals with different symmetries. In particular, the first quadrant $A B C$ in Fig. 6 is the domain of stability of the tetragonal phase $T_{3}$. This 'tetragonal sector' is partially overlapped by the two 'orthorhombic wedges' $D E F$ and $G H I$, shaded in Fig. 6, where the phases with symmetries $O_{1,2,3}$ and $O_{3,1 \pm 2}$, respectively, are stable. The domain where the $M_{3}$ phase is stable, computed from the system (5.19), is below the curve $P Q R$, and it overlaps the stability domains of the three other phases. Fig. 6 shows that in addition to four single-phase regions, there exist five two-phase regions. The most interesting feature of the diagram is the presence of two three-phase regions $E D^{\prime} P F^{\prime}$ and $H I^{\prime} R G^{\prime}$, where there is coexistence and metastability of the phase associations $T_{3}+O_{1,2,3}+M_{3}$ and $T_{3}+O_{3,1 \pm 2}+M_{3}$, respectively.

We remark that the domains of metastable phase coexistence are not usually indicated in the conventional thermodynamic phase diagrams like the one in Fig. 1, which only report as phase boundaries the 'Maxwell lines' separating the domains where the structure of the global energy minimizer changes. In our $\left(G_{\varepsilon}, G_{\delta}\right)$-diagram there are five Maxwell lines that meet at two t-o-m triple points; each triple point is located inside one of the three-phase metastability regions mentioned above. The explicit equations for the Maxwell lines can be obtained directly from the definition, and need not be made explicit 
here. Notice that because the coefficients $K_{\delta}$ and $K_{\delta}$ were chosen equal, the diagram in Fig. 6 is symmetric about the diagonal of the first quadrant; a more generic choice of coefficients with the above sign restrictions causes distortions of the diagram that do not affect its general topology ${ }^{7}$ (see [TD96] for a survey of different topologies compatible with the energy (5.3)).

In order to transform the formal $\left(G_{\varepsilon}, G_{\delta}\right)$-phase diagram into the physical $(p, T)$ diagram, we must relate the moduli $G_{\varepsilon}$ and $G_{\delta}$ to the environmental parameters $p$ and $T$. A linear pressure dependence is specified by (5.4); we prescribe the temperature dependence of $G_{\varepsilon}$ and $G_{\delta}$ by assuming that the elastic moduli of the tetragonal phase depend linearly on $T$. As $C_{11}-C_{12}$ is the modulus that softens, we set

$$
C_{11}-C_{12}=A_{\varepsilon}\left(T-T_{\varepsilon}\right), \quad C_{66}=A_{\delta} T+B_{\delta},
$$

so that from (5.4) we obtain

$$
G_{\varepsilon}=A_{\varepsilon}\left(T-T_{\varepsilon}\right)-\frac{H_{3}}{M} p, G_{\delta}=A_{\delta} T+B_{\delta}-\frac{H_{6}}{M} p
$$

For the tetragonal phase to be stable at high temperatures and zero pressure we require

$$
A_{\varepsilon} \geq 0, \quad A_{\delta} \geq 0
$$

Now, the experimental t-o-m phase diagram of $\mathrm{ZrO}_{2}$ in Fig. 1 shows that $\mathrm{t}-\mathrm{m}$ and $\mathrm{o}-\mathrm{m}$ Maxwell lines in the $(T, p)$-plane have negative slopes, while the t-o line has a slightly positive slope. From these conditions, together with (5.4), (5.25) and the fact that the bulk modulus $M$ is positive, we find the following restrictions on the coupling parameters $H_{3}$ and $H_{6}$ in (4.21):

$$
H_{3} \geq 0, \quad H_{6} \leq 0
$$

In fact, as the t-o line is almost parallel to the pressure axis, we have that $A_{\delta}$ and $H_{3}$ are almost zero, which means that the $C_{66}$ modulus may be considered temperature independent and that the dilatation $y_{1}$ is coupled to the order parameter $y_{6}$ but not to $y_{3}$. Due to (5.4)

\footnotetext{
7 We remark that with $\tilde{L}$ going to zero the two triple points get closer, and the orthorhombic wedges eventually overlap. This creates a four-phase domain in which all the four phases are metastable. For $\tilde{L}=0$ (no order-parameter coupling) the two triple points coincide, creating a non-generic (and nonGibbsian) quadruple point.
} 
and (5.21), we also see that the order-parameter coupling coefficient $L$ in (4.21) must be (sufficiently) negative

$$
L \leq \frac{1}{2} \frac{H_{3} H_{6}}{M} \leq 0
$$

In view of (5.15) and (5.18), similar bounds, which need not be detailed here, can be obtained for the coefficients $D_{3}$ and $D_{6}$ in (4.21). This completes the determination of the set of restrictions on the energy coefficients, which guarantee the existence of the t-o-m triple point in the suitable range of temperatures and pressures as in the experimental diagram of Fig. 1. In fact, under the assumptions formulated above, only the 'correct' triple point $T_{3}+O_{1,2,3}+M_{3}$ remains in the conventional part of the $p-T$ phase diagram; the other triple point maps into the half plane where $p<0$, and may possibly be observed at tensile stresses and high temperatures. The result of a suitable mapping $\left(G_{\varepsilon}, G_{\delta}\right) \leftrightarrow(T, p)$, as in (5.24) is shown in Fig. 7. In this paper, being mostly interested in the demonstration of the existence of the triple point, we did not try to obtain the energy parameters that best fit the available experimental data on zirconia. However, we notice that even within our simplified choice of the Landau parameters, Fig. 7 reproduces well the experimental diagram in Fig. 1, predicting the correct relation among the slopes for the equilibrium phase boundaries. What is new in the phase diagram of Fig. 7, comparing to the one presented in Fig. 1, is the indication of the two and three-phase metastability regions. In particular, we remark that the three-phase coexistence domain, within which lies the traditional isolated triple point, has a finite area.

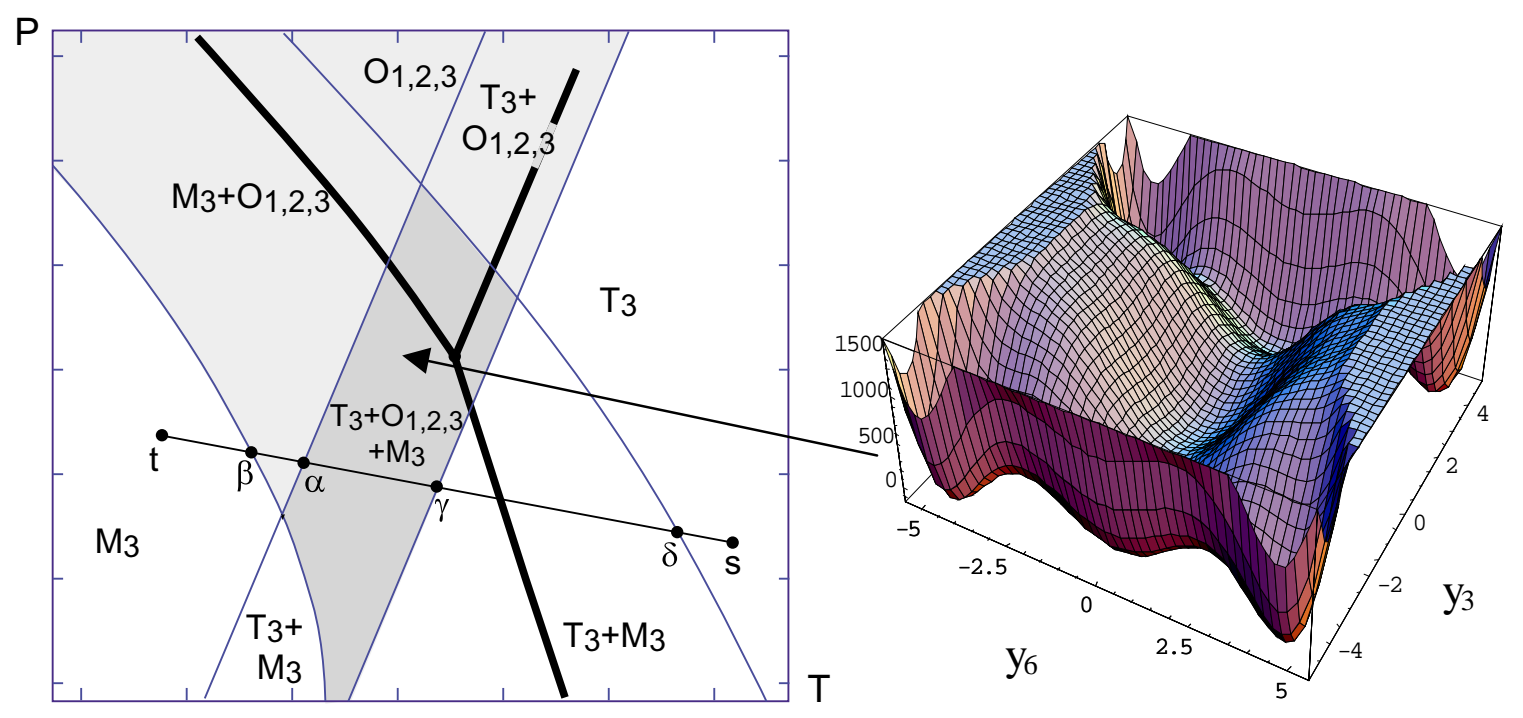


Fig. 7. Phase diagram in the ( $T, p)$-plane, showing the stability boundaries, the Maxwell lines (thick lines), and the $T_{3}+O_{1,2,3}+M_{3}$ triple point (compare with Fig. 1). The straight line $t-s$ is the same path as in Fig. 6, whose bifurcations are shown in Fig. 8. The insert shows the Landau energy surface, illustrating the variety of wells existing in the three-phase coexistence region, highlighted in gray.

To illustrate the nature of such a three-phase region, in Figs. 6 and 7 we show the energy landscape in the order-parameter space $y_{3}, y_{6}$, for $p$ - $T$ conditions near the triple point. In this region the Landau energy surface exhibits a total of seven wells, arranged in three coexisting variant structures as in (2.8); the wells all have the same depth at the triple point. As the environmental conditions are varied, these critical points with different symmetries describe branches that are connected through a variety of bifurcation patterns. In Figs. 6 and 7 we show a specific path $s-\delta-\gamma-\alpha-\beta-t$ in the $p-T$ plane, along which we compute the corresponding bifurcation diagram (compare with Fig. 8); this shows the sequence of t-o-m bifurcations and illustrates the double-bifurcation mechanism we propose for the well-known t-m transformation of zirconia. From Fig. 8, we see that the tetragonal and monoclinic branches are not directly connected, as we have two subcritical bifurcations: t-o at the point $\alpha$, and o-m at the point $\beta$ (with turning points $\gamma$ and $\delta$ ). We remark that the first bifurcation and the associated modulus softening is a consequence of the temperature dependence explicitly assumed for the tetragonal modulus $C_{11}-C_{12}$ in (5.23). The second bifurcation, on the other hand, is an effect of the internal coupling between the order parameters.

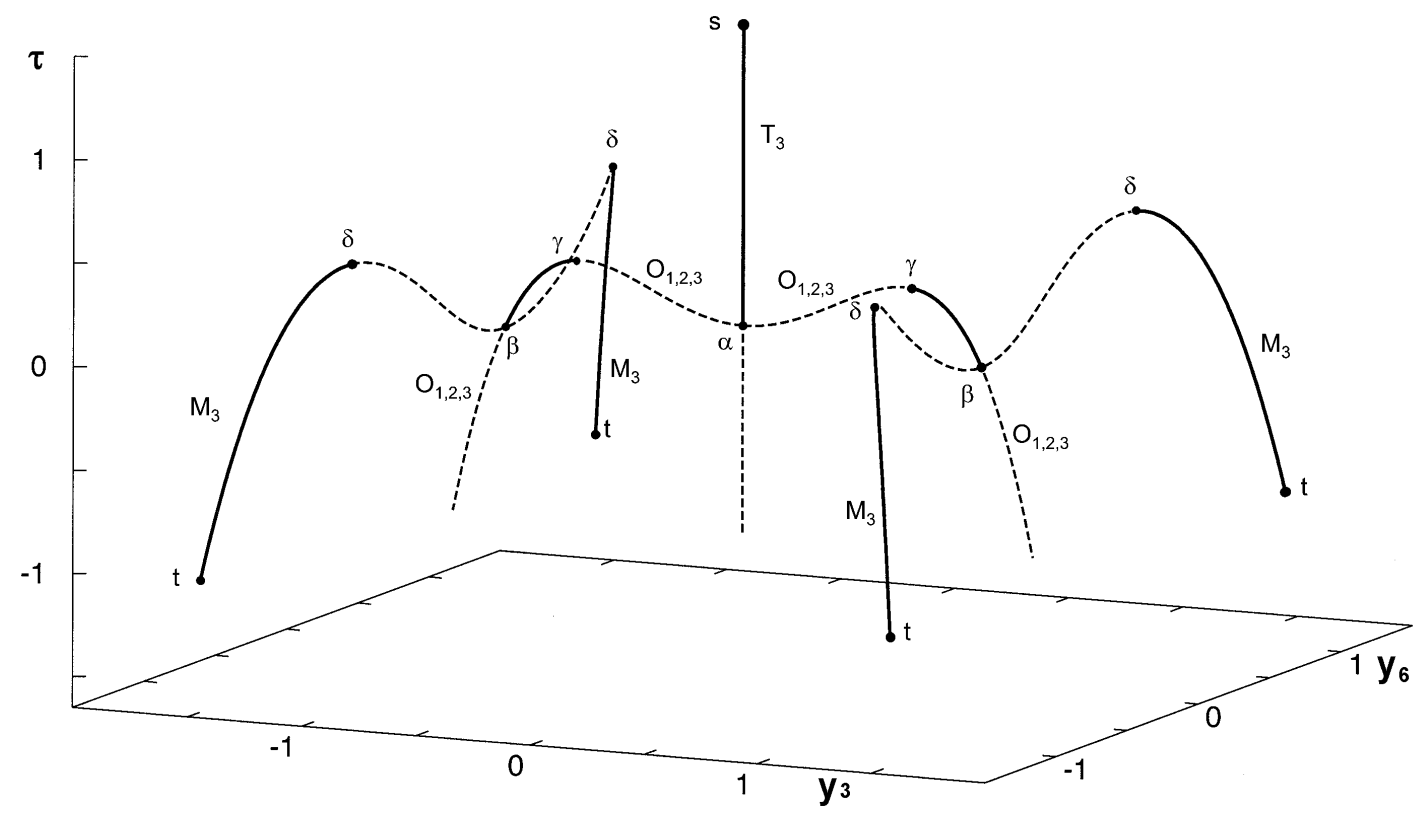


Fig. 8. T-o-m bifurcation diagram. The figure shows the bifurcation pattern along the path indicated by the straight line $t$-s in Figs. 6 and 7, to which the rest of the lettering refers; the parameter along this line is $\tau$ (our vertical axis here) growing from $t$ to $s$. The solid and dotted lines indicate respectively the (meta)stable and unstable portions of the t-o-m equilibrium branches.

\section{Conclusions}

In this paper we study the energy landscape for a class of elastic crystals exhibiting, under the combined influence of temperature and pressure, a 't-o-m' sequence of spontaneous symmetry-breaking phase transformations. We have constructed the minimal Landau-type energy function displaying a complete set of local and global minima with the desired symmetries and showing a triple point in its phase diagram. The preliminary analysis made in Section 2 of the t-o-m transformation mechanisms is necessary for the interpretation of the available experimental data on zirconia against a background in which no kinematic possibility is a priori excluded. As a result, we propose for this material a new transformation mechanism that is different from those previously considered in the literature, and which is based on a different modulus-softening hypothesis (double-bifurcation mechanism for the $t-m$ transformation). More data is needed to conduct a direct comparison of this prediction with experiment.

By using the explicit expression for the energy we have determined the existence and stability domains for the different phases of the t-o-m crystal with a triple point. Specifically, in addition to the classical equilibrium Maxwell lines, which intersect at the triple point and mark the boundaries of stability domains for the global minimizers, we have computed the co-existence domain boundaries for the different sets of metastable phases. For the singlecomponent t-o-m crystal, our analysis indicates the location of five two-phase and two threephase metastability domains (see Fig. 6 and footnote 7). In the latter the energy exhibits a total of seven energy wells belonging to three t-o-m sets of symmetry-related configurations.

We emphasize the importance of knowing the location of the domains with a maximum number of phases. Inside such domains the different energy wells may have nonequal depths; as a result they stretch beyond the conventional triple points where all the wells are at the same level. The occurrence of these metastable phase equilibria not accounted for by Gibbs' phase rule may help to understand some hysteretic processes in active crystals where metastability plays a major role. It may also help in the production of materials with the maximal flexibility of the microstructure; an interesting problem is thus to obtain criteria for the design of crystals whose phase diagrams exhibit triple-phase domains in desirable locations. Two ways can be envisaged to displace a triple point or a 'triple-area' in a phase diagram: (a) by selecting specific compositions for suitable alloys, and (b) by means of non-hydrostatic stresses.

The later issue is particularly relevant for the technological applications of zirconia. Thus a typical zirconia particle in a transformation-toughened ceramic undergoes a phase change near the tip of a crack; the corresponding non-hydrostatic conditions cannot be depicted in a conventional $p-T$ phase diagram. It is also known that by imbedding transforming inclusions into elastic matrices and thus by loading them through a combination of hard and soft devices, one can stabilize otherwise unstable phases with much advantage for the applications (e.g. [BT93]). All this suggests the necessity of studying 
systematically non-hydrostatic phase diagrams, which lie outside the scope of classical thermodynamical methods. On the contrary, our approach is perfectly suitable for this type of analysis (see also [ST96]). In particular, by using our energy function one can study shearinduced distortions of the conventional $p-T$ diagram, including the shifting in the location of the metastable triple areas.

Finally, we remark that in order to obtain a quantitatively sound model of $\mathrm{ZrO}_{2}$, our analysis should be extended to cover equilibrium configurations with more than three polymorphs and more than one triple point; in fact, no model of zirconia can be considered adequate unless the high-temperature face-centered cubic phase is also taken into account. Regarding the relevance of cubic zirconia, we notice that in the neighborhood of the (possibly unstable) cubic configuration of $\mathrm{ZrO}_{2}$, there exist three symmetry-related variants of the tetragonal phase, only one of which is considered in this paper. If such three tetragonal variants are taken into account, we actually obtain in the vicinity of the t-o-m triple point a total of 21 energy wells.

Acknowledgments The work of L.T. was supported by the NSF grant DMS-9803572; G.Z. acknowledges partial support of the Italian Cofinanziamento MURST 98 'Modelli matematici per la scienza dei materiali', and the Italian GNFM. We thank the IMA (Minneapolis, MN, USA) and each other's Institutions for the hospitality provided during much of our work. We thank Dr. A. Poliakov and Dr. G. Fadda for helpful discussions and their assistance with the computations and figures. Finally, we thank a referee for helpful comments and useful references.

\section{References}

[B15] Born, M. 1915; Dynamik der Krystallgitter. Teubner, Leipzig/Berlin.

[BJ87] Ball J. M., James R. D. 1987: “Finite phase mixtures as minimizers of energy”, Arch. Rat. Mech. Anal., 100 ,13-52.

[BJ92] Ball J.M., James R.D. 1992: "Proposed experimental test of a theory of fine microstructure and the two-well problem", Phil. Trans. Royal Soc. Lond. A338, 389 - 450.

[BJ00] Ball J.M., James R.D. 1998: The mathematics of microstructure, in preparation.

[BFJK94] Bhattacharya K., Firoozye N., James R., Kohn R. 1994: "Restrictions on Microstructure", Proc. Roy. Soc. Edinb, A124, 843-878.

[BFV91] Boysen, H., Frey, F., Vogt, T., 1991: "Neutron powder investigation of the tetragonal to monoclinic phase transformation in undoped zirconia", Acta Cryst. B47, 881-886.

[BH54] Born M., and Huang K. 1954; Dynamical theory of crystal lattices. Clarendon Press, Oxford.

[BHL83] Budiansky B., Hutchinson J., Lambropoulos J. 1983: "Continuum model of dilatant transformation toughening materials", J. Mech. Phys. Solids, 19, 337-356.

[BJP85] Block S., Da Jornada J.A.H., Piermarini G. J. 1985: "Pressure-temperature phase diagram of zirconia", J. Am. Ceram. Soc. 68, 497 - 499.

[BLL99] Bhattacharya K., Li B., Luskin M. 1999: "The simply laminated microstructure in martensitic crystals that undergo a cubic to orthorhombic phase transformation", Arch.Rat. Mech.Anal., 149, $123-154$.

[BT93] Budiansky B., Truskinovsky L. 1993: "On the mechanics of a stress induced transformation in zirconia", J. Mech. Phys. Solids 41, 1445 - 1459.

[Cha88] Chan S.K. 1988: "The polymorphic transformation of zirconia", Physica B150, 212 - 222.

[Chr75] Christian J.W. 1975: The theory of transformations in metals and alloys, OUP, Oxford.

[CK88] Chipot M and Kinderlehrer D 1988; "Equilibriun configurations of crystals", Arch. Rat. Mech. Anal. 103 237-277

[DL98] Dewhurst, J.K., Lowther, J.E., 1998: "Relative stability, structure, and elastic properties of several phases of pure zirconia", Phys. Rev. B57, 741-747. 
[DF80] Dunn J.E., Fosdick R.L. 1980: "The morphology and stability of material phases", Arch. Rat. Mech. Anal. 74, 1-99.

[E70] Ericksen J.L. 1970: "Nonlinear elasticity of diatomic crystals”, Int. J. solids Structures 6, 951 - 957.

[E80] Ericksen J.L. 1980: "Some phase transitions in crystals", Arch. Ration. Mech. Anal. 73, 99 - 124.

[E89] Ericksen J.L. 1989: "Weak martensitic transformations in Bravais lattices", Arch. Ration. Mech. Anal. 107, 23 - 36.

[E93] Ericksen J. L. 1993: "Local bifurcation theory for thermoelastic Bravais lattices", in Microstructure and phase transitions, Lecture Notes in Physics 344, Springer, Berlin, etc.

[E99] Ericksen J.L. 1999: "Notes on the X-ray theory", J. Elasticity, 55, 201 - 218.

[FBV90] Frey, F., Boysen, H., Vogt, T. 1990: "Neutron powder investigation of the monoclinic to tetragonal phase transformation in undoped zirconia", Acta Cryst. B46, 724-730.

[FPF00] Fabris S., Paxton A.T., Finnis M.W. 2000: "Relative energetics and structural properties of zirconia using a self-consistent tight-binding model”, Phys. Rev. B61, 6617-6630

[GHS89] Green D.J., Hannink R.H.J., Swain M.V. 1989: Transformation toughening of ceramics, CRC Press, Boca-Raton.

[HKRH90] Howard, C.J., Kisi, E.H., Roberts, R.B., Hill, R.J. 1990: "Neutron diffraction studies of phase transformations between tetragonal and orthorhombic zirconia in magnesia-partially-stabilized zirconia", J. Am. Ceram. Soc., 73, 2828-2833.

[HM90] Hugo, G.R., Muddle, B.C., 1990: "The tetragonal to monoclinic transformation in ceria-zirconia", Materials Science Forum, 56-58, 357-362.

[ID89] Ishibashi, Y., Dvorak, V., 1989: "A phenomenological theory of structural phase transitions in zirconia”, J. Phys. Soc. Japan, 58, 4211-4214.

[IS90] Izyumov Y.A., Syromyatnikov V.N. 1990: Phase transitions and crystal symmetry, Kluwer, Dordrecht, etc.

[JH00] James R. D., Hane K. F. 2000: "Martensitic transformations and shape-memory materials", Acta Materialia 48, 197 - 222.

[Kh83] Khachaturyan A. 1983: Theory of structural transformations in solids, Wiley, New York.

[Ki98] Kisi E. 1998: Zirconia engineering ceramics: old challenges - new ideas (E. Kisi, ed.), Uetichon, Zuerich, etc.

[KHH89] Kisi E.H., Howard C.J., Hill, R.J. 1989: “Crystal structure of orthorhombic zirconia in partially stabilized zirconia”, J. Am. Ceram. Soc., 72, 1757-1760.

[KPA88] Kudoh Y., Prewitt C. T., Arashi H. 1989: Premonitory twinning in the high pressure phase of $\mathrm{ZrO}_{2}$, in Annual Report of the Director, Geophysical Laboratory, Carnegie Institution, Washington D.C.

[La37] Landau L.D. 1937: "On the theory of phase transitions", in Collected papers of L. D. Landau, Gordon and Breach, New York, etc.

[Lo27] Love A.E.H. 1927: A treatise on the mathematical theory of elasticity, Dover, New York.

[Lu96] Luskin M. 1996: "On the computation of crystalline microstructure", Acta Num. 5, 191 - 258.

[LL86] Landau L.D., Lifchitz E. M. 1986, Theory of elasticity, Pergamon Press, Oxford.

[LTAP93] Leger J.M., Tomaszewski P.E., Atouf A., Pereira A.S. 1993: "Pressure-induced structural phase transitions in zirconia under high pressure", Phys. Rev. B47, 14075 - 14083.

[M98] Müller S. 1998: Variational models for microstructure and phase transitions, Lecture notes n. 2, Max Planck Institute, Leipzig.

[OM98] Ondik H. M., McMurdie H. F. 1998: Phase diagrams for zirconium and zirconia systems, Am. Cer. Soc., Westerville, OH, USA.

[P84] Pitteri M. 1984: "Reconciliation of global and local symmetries in crystals", J. Elasticity 14, 175 190.

[OYKIN91] Ohtaka O., Yamanaka T., Kume S., Ito E., Navrotsky A. 1991: "Stability of monoclinic and orthorhombic zirconia: studies by high-pressure phase equilibria and calorimetry", J. Am. Ceram. Soc. 74, 505 - 509 .

[PZ00] Pitteri M., Zanzotto G. 2000: Continuum models for phase transitions and twinning in crystals, Chapman and Hall, London, to appear.

[R78] Roytburd A.L. 1978: "Martensitic transformations as a typical phase transformation in solids", Solid State Physics 33, 317 - 352.

[Si97] Simha N. 1997: "Twin and habit plane microstructures due to the tetragonal-to-monoclinic transformation of zirconia”, J. Mech. Phys. Solids 45, 261 - 292. 
[St50] Stakgold, I. 1950; "The Cauchy relations in a molecular theory of elasticity", Quart. Appl. Math., 8, 169-186.

[SD86] Salje E.K.H., Devarajan V. 1986: "Phase transitions in systems with strain-induced coupling between two order parameters", Phase Trans. 6, 253 - 248.

[SM58] Smith G.F., Rivlin R.S. 1958: "The strain-energy function for anisotropic elastic materials", Trans. Amer. Math. Soc. 88, 175 - 193.

[SMS74] Subbarao E.C., Maiti H.S., Srivastava K.K. 1974: "Martensitic transformations in zirconia", Phys. Stat. Sol. 21, 9 - 40.

[ST96] Simha N., Truskinovsky L. 1996: "Phase diagram of zirconia in stress space", in Contemporary research on the mechanics and mathematics of materials, R. C. Batra and M. F. Beatty eds., CIMNE, Barcelona.

[TD96] Toledano P., Dmitriev V. 1996: Reconstructive phase transitions in crystals and quasicrystals, World Scientific, Singapore, etc.

[W64] Wayman C.M. 1964: Introduction to the theory of martensitic transformations, Macmillan, New York, etc.

[WLR53] Wechsler M.S., Liebermann D.S., Reed T.A. 1953: "On the theory of the formation of martensite", Trans. AIME J.Metals 197, $1503-1515$.

[WP98] Wu X. C, Pence . T.J. 1998: "Two variant modeling of shape memory materials: unfolding a phase diagram triple point", J. Intelligent Material Systems and Structures, 9, 335-354.

[Z96a] Zanzotto G. 1996: "The Cauchy-Born hypothesis, nonlinear elasticity, and mechanical twinning in crystals", Acta Cryst. A52, 839 - 849.

[Z96b] Zanzotto G. 1996: "Weak and symmetry-breaking phase transformations in simple lattices", preprint. 